\title{
Well-posedness of Stochastic Riccati Equations and Closed-Loop Solvability for Stochastic Linear Quadratic Optimal Control Problems
}

\author{
Qi L $\ddot{u ̈}^{*}$
}

\begin{abstract}
We study the closed-loop solvability of a stochastic linear quadratic optimal control problem for systems governed by stochastic evolution equations. This solvability is established by means of solvability of the corresponding Riccati equation, which is implied by the uniform convexity of the quadratic cost functional. At last, conditions ensuring the uniform convexity of the cost functional are discussed.
\end{abstract}

2010 Mathematics Subject Classification. 93E20, 49N10, 49N35.

Key Words. stochastic linear quadratic control problem, stochastic evolution equation, closed-loop solvability, Riccati equation.

\section{Introduction}

Let $(\Omega, \mathcal{F}, \mathbb{F}, \mathbb{P})$ be a complete filtered probability space on which a standard one-dimensional Brownian motion $W(\cdot)=\{W(t)\}_{t \geq 0}$ is defined, and $\mathbb{F}=\left\{\mathcal{F}_{t}\right\}_{t \geq 0}$ is the natural filtration of $W(\cdot)$ augmented by all the $\mathbb{P}$-null sets in $\mathcal{F}$.

Let $T>0$ be a fixed time horizon. For any $t \in[0, T)$ and Banach space $\mathbb{H}$, let

$$
\begin{gathered}
L_{\mathcal{F}_{t}}^{2}(\Omega ; \mathbb{H})=\left\{\xi: \Omega \rightarrow \mathbb{H} \mid \xi \text { is } \mathcal{F}_{\left.t^{-} \text {strongly measurable, } \mathbb{E}|\xi|_{\mathbb{H}}^{2}<\infty\right\},}\right. \\
L_{\mathbb{F}}^{2}(t, T ; \mathbb{H})=\{\varphi:[t, T] \times \Omega \rightarrow \mathbb{H} \mid \varphi(\cdot) \text { is } \mathbb{F} \text {-progressively measurable, } \\
\left.\qquad \mathbb{E} \int_{t}^{T}|\varphi(s)|_{\mathbb{H}}^{2} d s<\infty\right\}, \\
C_{\mathbb{F}}\left([t, T] ; L^{2}(\Omega ; \mathbb{H})\right)=\left\{\varphi:[t, T] \times \Omega \rightarrow \mathbb{H} \mid \varphi(\cdot) \text { is } \mathbb{F} \text {-adapted, } \varphi:[t, T] \rightarrow L_{\mathcal{F}_{T}}^{2}(\Omega ; \mathbb{H})\right. \\
\text { is continuous, }\},
\end{gathered}
$$

${ }^{*}$ School of Mathematics, Sichuan University, Chengdu, 610064, China. This work is supported the NSF of China under grant 11471231, the Fundamental Research Funds for the Central Universities in China under grant 2015SCU04A02, the NSFC-CNRS Joint Research Project under grant 11711530142 and Grant MTM2014-52347 of the MICINN, Spain. 


$$
\begin{gathered}
L_{\mathbb{F}}^{2}\left(\Omega ; L^{1}(t, T ; \mathbb{H})\right)=\{\varphi:[t, T] \times \Omega \rightarrow \mathbb{H} \mid \varphi(\cdot) \text { is } \mathbb{F} \text {-progressively measurable }, \\
\left.\mathbb{E}\left(\int_{t}^{T}|\varphi(s)|_{\mathbb{H}} d s\right)^{2}<\infty\right\} .
\end{gathered}
$$

Let $\mathbb{H}_{1}$ and $\mathbb{H}_{2}$ be two Banach spaces. Denote by $\mathcal{L}\left(\mathbb{H}_{1} ; \mathbb{H}_{2}\right)$ (resp. $\left.\mathcal{L}\left(\mathbb{H}_{1}\right)\right)$ the set of all bounded linear operators from $\mathbb{H}_{1}$ to $\mathbb{H}_{2}\left(\right.$ resp. $\left.\mathbb{H}_{1}\right)$. If $\mathbb{H}$ is a Hilbert space, then we set

$$
\mathbb{S}(\mathbb{H}) \triangleq\{F \in \mathcal{L}(\mathbb{H}) \mid F \text { is symmetric }\},
$$

and

$$
\overline{\mathbb{S}_{+}}(\mathbb{H}) \triangleq\{F \in \mathbb{S}(\mathbb{H}) \mid\langle F \xi, \xi\rangle \geq 0, \quad \forall \xi \in H\} .
$$

Here and in what follows, for simplicity of notations, when there is no confusion, we shall use $\langle\cdot, \cdot\rangle$ for inner products in possibly different Hilbert spaces.

For any interval $\left[t_{1}, t_{2}\right] \subset[0,+\infty)$, denote by $C\left(\left[t_{1}, t_{2}\right] ; \mathbb{S}(\mathbb{H})\right)$ the set of all continuous mappings from $\left[t_{1}, t_{2}\right]$ to $\mathbb{S}(\mathbb{H})$, which is a Banach space with the norm

$$
|F|_{C\left(\left[t_{1}, t_{2}\right] ; \mathbb{S}(\mathbb{H})\right)} \triangleq \sup _{t \in\left[t_{1}, t_{2}\right]}|F(t)|_{\mathcal{L}(\mathbb{H})} .
$$

Denote by $C_{\mathcal{S}}\left(\left[t_{1}, t_{2}\right] ; \mathbb{S}(\mathbb{H})\right)$ the set of all strongly continuous mappings $F:\left[t_{1}, t_{2}\right] \rightarrow$ $\mathbb{S}(\mathbb{H})$, that is, $F(\cdot) \xi$ is continuous on $\left[t_{1}, t_{2}\right]$ for each $\xi \in H$. Let $\left\{F_{n}\right\}_{n=1}^{\infty} \subset C_{\mathcal{S}}\left(\left[t_{1}, t_{2}\right] ; \mathbb{S}(\mathbb{H})\right)$. We say $\left\{F_{n}\right\}_{n=1}^{\infty}$ converges strongly to $F \in C_{\mathcal{S}}\left(\left[t_{1}, t_{2}\right] ; \mathbb{S}(\mathbb{H})\right)$ if

$$
\lim _{n \rightarrow \infty} F_{n}(\cdot) \xi=F(\cdot) \xi, \quad \forall \xi \in \mathbb{H} \text {. }
$$

In this case, we write

$$
\lim _{n \rightarrow \infty} F_{n}=F \quad \text { in } C_{\mathcal{S}}\left(\left[t_{1}, t_{2}\right] ; \mathbb{S}(\mathbb{H})\right) .
$$

If $F \in C_{\mathcal{S}}\left(\left[t_{1}, t_{2}\right] ; \mathbb{S}(\mathbb{H})\right)$, then, by the Uniform Boundedness theorem, the quantity

$$
|F|_{C_{\mathcal{S}}\left(\left[t_{1}, t_{2}\right] ; \mathbb{S}(\mathbb{H})\right)} \triangleq \sup _{t \in\left[t_{1}, t_{2}\right]}|F(t)|_{\mathcal{L}(\mathbb{H})}
$$

is finite, and $C_{\mathcal{S}}\left(\left[t_{1}, t_{2}\right] ; \mathbb{S}(\mathbb{H})\right)$ is a Banach space with this norm (see [4] for the proof).

Let $H$ and $U$ be two separable Hilbert spaces. Consider the following controlled linear stochastic evolution equation (SEE, for short):

$$
\left\{\begin{array}{l}
d x=\left[\left(A+A_{1}\right) x+B u\right] d s+(C x+D u) d W(s) \quad \text { in }(t, T], \\
x(t)=\eta \in H
\end{array}\right.
$$

where $A$ generates a $C_{0}$-semigroup $\left\{e^{A s}\right\}_{s \geq 0}$ on $H$,

$$
\left\{\begin{array}{l}
A_{1}(\cdot) \in L^{1}(0, T ; \mathcal{L}(H)), \quad B(\cdot) \in L^{2}(0, T ; \mathcal{L}(U ; H)), \\
C(\cdot) \in L^{2}(0, T ; \mathcal{L}(H)), \quad D(\cdot) \in L^{\infty}(0, T ; \mathcal{L}(H ; U))
\end{array}\right.
$$

In the above, $x(\cdot)$ is the state process, and $u(\cdot) \in \mathcal{U}[t, T] \triangleq L_{\mathbb{F}}^{2}(t, T ; U)$ is the control process. Any $u(\cdot) \in \mathcal{U}[t, T]$ is called an admissible control (on $[t, T]$ ). For any initial pair $(t, \eta) \in$ 
$[0, T) \times H$ and admissible control $u(\cdot) \in \mathcal{U}[t, T],(1.1)$ admits a unique mild solution $x(\cdot) \equiv$ $x(\cdot ; t, \eta, u(\cdot))$ (see Lemma 3.11). Here and in what follows, to simplify the notations, the time variable $s$ is suppressed in $B, C$, etc.

Next we introduce the following cost functional:

$$
\mathcal{J}(t, \eta ; u(\cdot)) \triangleq \mathbb{E}\langle G x(T), x(T)\rangle+\mathbb{E} \int_{t}^{T}(\langle Q x, x\rangle+\langle R u, u\rangle) d s
$$

where

$$
G \in \mathbb{S}(H), \quad Q(\cdot) \in L^{1}(0, T ; \mathbb{S}(H)), \quad R(\cdot) \in L^{\infty}(0, T ; \mathbb{S}(U)) .
$$

The optimal control problem studied in this paper is as follows.

Problem (SLQ). For any given initial pair $(t, \eta) \in[0, T) \times H$, find a $\bar{u}(\cdot) \in \mathcal{U}[t, T]$, such that

$$
V(t, \eta) \triangleq \mathcal{J}(t, \eta ; \bar{u}(\cdot))=\inf _{u(\cdot) \in \mathcal{U}[t, T]} \mathcal{J}(t, \eta ; u(\cdot))
$$

Any $\bar{u}(\cdot) \in \mathcal{U}[t, T]$ satisfying (1.3) is called an optimal control of Problem (SLQ) for the initial pair $(t, \eta)$, and the corresponding $\bar{x}(\cdot) \equiv x(\cdot ; t, \eta, \bar{u}(\cdot))$ is called an optimal state process; the pair $(\bar{x}(\cdot), \bar{u}(\cdot))$ is called an optimal pair. The function $V(\cdot, \cdot)$ is called the value function of Problem (SLQ).

Remark 1.1 In this paper, we assume that $B(\cdot) \in L^{2}(0, T ; \mathcal{L}(U ; H))$ and $D(\cdot) \in L^{\infty}(0, T$; $\mathcal{L}(H ; U))$. Thus, our results can only be applied to controlled stochastic partial differential equations with distributed controls. To study systems with boundary controls, one needs to make some further assumptions, such as the semigroup $\left\{e^{A s}\right\}_{s \geq 0}$ has some smoothing effect. With such assumptions, we can prove Theorem 2.2(the details are lengthy and beyond the scope of this paper). However, since such assumptions contradict (AS2) given below, under them, we do not know how to prove Theorem [2.1. Fortunately, there are many controlled stochastic partial differential equations satisfying these conditions, such as stochastic wave (resp. Schrödinger, KdV, transport, plate) equations with internal controls.

Remark 1.2 In this paper, we assume that the coefficients are deterministic. In such case, the corresponding Riccati equation (2.2) is an operator-valued deterministic differential equation. If one considers the problem that the coefficients are stochastic, then an operator-valued backward stochastic differential equation should be studied. Until now, only some very special cases of such equations are investigated (e.g.[13, 14, 22]]).

The study of an optimal control problem for a linear system with a quadratic cost functional (LQ problem, for short) dates back at least to [3], in which the system is governed by a linear ordinary differential equation. It an be regarded as the simplest nontrivial optimal control problems, namely, the system is linear and the cost functional is quadratic. Consequently, it has elegant and fruitful mathematical structure. Furthermore, it has important applications (e.g. [2]). Such kind of problem was investigated extensively in the literature for a variety of deterministic systems(e.g. [2, 20]).

LQ problems for controlled stochastic differential equations (SDEs for short) was first studied in [36]. Such problems are the most important examples of the stochastic control 
problems, especially in their applications in finance and economics. There are a huge amount works addressing the LQ problems for controlled SDEs(see [6, 8, 9, 17, 15, 26, 27, 31, 37, 38] and the rich references therein).

SEEs are used to describe a lot of random phenomena appearing in physics, chemistry, biology, and so on. In many situations SEEs are more realistic mathematical models than the deterministic ones (e.g. [7, 18, 19]). Thus, there are many works addressing the optimal control problems for SEEs. In particular, we refer the readers to [1, 10, 11, 13, 14, 16, 32, 33] and the rich references therein for LQ problem for controlled SEEs.

In those works, the following assumption was taken for granted:

$$
G \geq 0, \quad R(s) \geq \delta I, \quad Q(s) \geq 0, \quad \text { a.e. } s \in[t, T],
$$

for some $\delta>0$. Under (1.4), when all the operators in the SEEs are deterministic, people had proven the corresponding Riccati equation is uniquely solvable and Problem (SLQ) admits a unique optimal control which has a linear state feedback representation (under certain technical conditions)(e.g. 32]). On the other hand, in the case that some operators in the SEEs are stochastic, Problem (SLQ) was well studied when $D=0$ and (1.4) holds (e.g. [13, 14]).

In [8], the authors discovered a new phenomenon, that is, Problem (SLQ) might still be solvable for controlled SDEs even if $R(s)$ is not positive definite for a.e. $s \in[0, T]$. This motivated many subsequent works concerning controlled SDEs (e.g. [9, 17, 21, 26, 29, 28, 37]). As far as we know, there is no generalization of this to controlled SEEs, which is one of the main purpose of this paper.

Recently, in [28, the authors introduced the notions of open-loop and closed-loop solvability of stochastic LQ problems and showed the difference between these two concepts. Roughly speaking, open-loop (resp. closed-loop) solvability of a stochastic LQ problem means that there is an open-loop (resp. closed-loop) optimal control of that problem. Another main purpose of this paper is to study the relationship between the closed-loop solvability for Problem (SLQ) and the unique solvability of the corresponding Riccati equation.

In view of the main novel contributions distinguishing this work from other publications in the literatures are: (1) $R(s) \geq \delta I$ may not hold; (2) the equivalence between the existence of an optimal feedback operator and the existence of regular solution to the stochastic Riccati equation is established. Although these two phenomena has been already discovered for stochastic LQ problem for controlled SDEs, one cannot simply mimic the method to solve our problem. There are some difficulties needing to be overcome. For example, in finite dimensional case, one can represent the solution to the Riccati equation by the product of a solution to a matrix-valued backward SDE and a solution to a matrix-valued SDE. In the infinite-dimensional case, formally, the matrix-valued SDE becomes an operatorvalued stochastic differential equation. Since there is no suitable integration theory for general operator-valued stochastic processes with respect to $W(\cdot)$ (see [34, 35] for the details), the solution to these operator-valued processes cannot be defined in the classical sense. More details on the difficulties arising in the infinite dimensional settings can be found in Sections 35.

The rest of the paper is organized as follows. In Section 2, we present the main results of this paper. Section 3 is devoted to giving some preliminary results. Sections 4 and 5 are addressed to the proofs of the main results. In Section 6, we discuss the the uniform 
convexity of the cost functional. At last, in the appendix, we give proofs for some preliminary results in Section 3 .

\section{Statements of the main results}

In this section, we present the main results of this paper. To begin with, let us first introduce some concepts.

Definition 2.1 We call $\bar{\Theta}(\cdot) \in L^{2}(t, T ; \mathcal{L}(U ; H))$ an optimal feedback operator of Problem $(S L Q)$ on $[t, T]$ if

$$
\mathcal{J}(t, \eta ; \bar{\Theta}(\cdot) \bar{x}(\cdot)) \leq \mathcal{J}(t, \eta ; u(\cdot)), \quad \forall \eta \in H, \quad u(\cdot) \in \mathcal{U}[t, T],
$$

where $\bar{x}(\cdot)$ is the mild solution to (1.1) with $u(\cdot)=\bar{\Theta}(\cdot) \bar{x}(\cdot)$.

Definition 2.2 Problem (SLQ) is said to be (uniquely) closed-loop solvable on $[t, T]$ if an optimal feedback operator (uniquely) exists on $[t, T]$.

Problem (SLQ) is said to be (uniquely) closed-loop solvable if it is (uniquely) closed-loop solvable on any time horizon $[t, T]$.

Remark 2.1 Clearly, if $\bar{\Theta}(\cdot)$ is an optimal feedback operator of Problem (SLQ) on [t,T], then the control $\bar{u}(\cdot) \equiv \bar{\Theta}(\cdot) \bar{x}(\cdot)$ is an optimal control of Problem (SLQ) for the initial pair $(t, \bar{x}(t))$. Hence, the closed-loop solvability of Problem (SLQ) implies the existence of an optimal control of that problem. However, the converse is untrue. An counterexample is given in [28] for controlled SDEs.

Next, let us recall the definition of the generalized pseudo inverse of a self-adjoint operator. More details and related proofs can be found in [5].

Definition 2.3 Let $H_{1}$ be a Hilbert space. For $F \in \mathcal{S}\left(H_{1}\right)$, a generalized pseudo inverse of $F$ is defined as a linear operator $F^{\dagger}: \mathcal{D}\left(F^{\dagger}\right) \rightarrow H_{1}$ satisfying the following four criteria:

$$
F F^{\dagger} F=F, \quad F^{\dagger} F F^{\dagger}=F^{\dagger}, \quad\left(F F^{\dagger}\right)^{*}=F F^{\dagger}, \quad\left(F^{\dagger} F\right)^{*}=F^{\dagger} F .
$$

When $F$ is injective, $F^{\dagger}$ is a left inverse of $F$. In this case, $F^{\dagger} F=I$. When $F$ is surjective, $F^{\dagger}$ is a right inverse, as $F F^{\dagger}=I$. When $F$ is self-adjoint, $F^{\dagger}$ always exists, which may be unbounded.

Next, we introduce the Riccati equation associated with Problem (SLQ) below:

$$
\left\{\begin{array}{l}
\dot{P}+P\left(A+A_{1}\right)+\left(A+A_{1}\right)^{*} P+C^{*} P C+Q-L^{*} K^{\dagger} L=0 \quad \text { in }[t, T), \\
P(T)=G,
\end{array}\right.
$$

where

$$
L(\cdot)=B(\cdot)^{*} P(\cdot)+D(\cdot)^{*} P(\cdot) C(\cdot), \quad K(\cdot)=R(\cdot)+D(\cdot)^{*} P(\cdot) D(\cdot) .
$$


Definition 2.4 We call $P \in C_{\mathcal{S}}([t, T] ; \mathbb{S}(H))$ a mild solution to (2.2) if for any $\eta \in H$ and $s \in[t, T]$,

$$
P(s) \eta=e^{A^{*}(T-s)} G e^{A(T-s)} \eta+\int_{s}^{T} e^{A^{*}(\tau-s)}\left(P A_{1}+A_{1}^{*} P+C^{*} P C+Q-L^{*} K^{\dagger} L\right) e^{A(\tau-s)} \eta d \tau .
$$

Now we can give the following notion.

Definition 2.5 A mild solution $P(\cdot) \in C([t, T] ; \mathbb{S}(H))$ of (2.2) is regular if

$$
\begin{gathered}
\mathcal{R}(L(s)) \subseteq \mathcal{R}(K(s)), \quad \text { a.e. } \quad s \in[t, T], \\
K(\cdot)^{\dagger} L(\cdot) \in L^{2}(t, T ; \mathcal{L}(H ; U)),
\end{gathered}
$$

and

$$
K(s) \geq 0, \quad \text { a.e. } s \in[t, T] .
$$

Definition 2.6 A solution $P(\cdot)$ of $(2.2)$ is strongly regular if

$$
K(s) \geq \lambda I, \quad \text { a.e. } s \in[t, T],
$$

for some $\lambda>0$.

Definition 2.7 The Riccati equation (2.2) is said to be (strongly) regularly solvable, if it admits a (strongly) regular solution.

Remark 2.2 As far as we know, the notion of (strongly) regular solution of a matrix-valued Riccati equation was first introduced in [28].

Remark 2.3 Clearly, condition (2.6) implies (2.3)-(2.5). Thus, a strongly regular solution $P(\cdot)$ is regular. Moreover, if $P$ is a strongly regular solution, then $K(s)$ is invertible for a.e. $s \in[0, T]$, i.e., the generalized pseudo inverse is the inverse of $K(s)$.

To investigate the relation between the closed-loop solvability of Problem (SLQ) and the existence of a regular solution to the Riccati equation (2.2), we need to make the following assumptions for $A$.

(AS1) $A$ generates a $C_{0}$-group on $H$.

(AS2) The eigenfunctions $\left\{e_{j}\right\}_{j=1}^{\infty}$ of $A$ constitutes an orthonormal basis of $H$.

Remark 2.4 We put two assumptions on $A$, i.e., A generates a $C_{0}$-group and its eigenfunctions constitutes an orthonormal basis of $H$. Both of them play important roles in the proof of Theorem 2.1. Indeed, in the proof of Theorem 2.1, we need to find a finite dimensional approximation of (2.2), i.e., we should approximate operator-valued processes by matrix-valued processes. To this end, $H$ should has an orthonormal basis $\left\{e_{j}\right\}_{j=1}^{\infty}$. This is true since $H$ is separable. However, for getting some good estimates (see Steps 1 and 2 in the proof of Theorem (2.1), we need the fact that $e_{j}$ is an eigenfunction of $A$ for each $j \in \mathbb{N}$. This leads to (AS2) Furthermore, to get the inverse of the operator $X(s, \cdot)$ given in step 2 in the proof of Theorem 2.1, we need (AS1). 
It seems that both (AS1) and (AS2) are only technical assumptions and can be dropped. However, we do not know how to do it now. For example, without (AS1), $-A^{*}$ does not generate a $C_{0}$-semigroup. Then the equation (3.7) is not well-posed and the operator $\widetilde{X}(s, \cdot)$ is not well defined. Then one cannot show the inverse of $X(s, \cdot)$ On the other hand, without (AS2), we cannot get (3.10) and (3.11), which are keys in the proof of many results, such as (4.8) and (4.10). More details can be see in the proof of Theorem 2.1.

Fortunately, under these conditions, the system (1.1) covers many controlled stochastic PDEs, such as stochastic wave equations, stochastic Schrödinger equations, stochastic beam equations, with internal controls.

Theorem 2.1 i) If the Riccati equation (2.2) admits a regular solution $P(\cdot) \in C_{\mathcal{S}}([t, T] ; \mathbb{S}(H))$, then Problem (SLQ) is closed-loop solvable. In this case, the optimal feedback operator $\bar{\Theta}(\cdot)$ is given by

$$
\bar{\Theta}=-K^{\dagger} L+\left(I-K^{\dagger} L\right) \theta,
$$

for some $\theta(\cdot) \in L^{2}(t, T ; \mathcal{L}(U ; H))$, and the value function is

$$
V(t, \eta)=\mathbb{E}\langle P(t) \eta, \eta\rangle \text {. }
$$

ii) Let (AS1) and (AS2) hold. If Problem (SLQ) is closed-loop solvable, then the Riccati equation (2.2) admits a regular solution $P(\cdot) \in C_{\mathcal{S}}([t, T] ; \mathbb{S}(H))$.

Next result gives a sufficient and necessary condition for the existence of a strongly regular solution to the Riccati equation (2.2).

Theorem 2.2 The following statements are equivalent:

(i) The map $u(\cdot) \mapsto \mathcal{J}(0,0 ; u(\cdot))$ is uniformly convex, i.e., there exists a $\lambda>0$ such that

$$
\mathcal{J}(0,0 ; u(\cdot)) \geq \lambda \mathbb{E} \int_{0}^{T}|u(s)|_{U}^{2} d s, \quad \forall u(\cdot) \in \mathcal{U}[0, T]
$$

(ii) The Riccati equation (2.2) admits a strongly regular solution $P(\cdot) \in C_{\mathcal{S}}([0, T] ; \mathbb{S}(H))$.

Remark 2.5 Clearly, if (1.4) holds, then the map $u(\cdot) \mapsto \mathcal{J}(0,0 ; u(\cdot))$ is uniformly convex. On the other hand, there are some interesting cases that the map $u(\cdot) \mapsto \mathcal{J}(0,0 ; u(\cdot))$ is uniformly convex but (1.4) does not hold. Please see Section 6 for the details.

Combining Theorems 2.1 and 2.2, we obtain the following corollary.

Corollary 2.1 Let (2.9) hold. Then, at any $(t, \eta) \in[0, T) \times H$, Problem (SLQ) admits a unique optimal control $\bar{u}(\cdot)$ of a state feedback form:

$$
\bar{u}(\cdot)=-K(\cdot)^{-1} L(\cdot) \bar{x}(\cdot),
$$

where $P(\cdot)$ is the unique strongly regular solution of (2.2) with $\bar{x}(\cdot)$ being the solution to the following closed-loop system:

$$
\left\{\begin{array}{l}
d \bar{x}=\left(A+A_{1}-B K^{-1} L\right) \bar{x} d s+\left(C-D K^{-1} L\right) \bar{x} d W(s) \quad \text { in }(t, T], \\
\bar{x}(t)=\eta
\end{array}\right.
$$

Proof: By Theorem 2.2, the Riccati equation (2.2) admits a unique strongly regular solution $P(\cdot) \in C([0, T] ; \mathbb{S}(H))$. Applying Theorem 2.1, we get the desired result. 


\section{Some preliminaries}

In this section, we present some useful results which will be used in the sequel. Except Lemma 3.5, the proofs of other results are put in the appendix.

For any $t \in[0, T)$, consider the following SEE:

$$
\left\{\begin{array}{l}
d x=[(A+\mathcal{A}) x+f] d s+(\mathcal{B} x+g) d W(s) \quad \text { in }(t, T], \\
x(t)=\eta
\end{array}\right.
$$

Here $\mathcal{A} \in L^{1}(t, T ; \mathcal{L}(H)), \mathcal{B} \in L^{2}(t, T ; \mathcal{L}(H)), \eta \in L_{\mathcal{F}_{t}}^{2}(\Omega ; H), f \in L_{\mathbb{F}}^{2}\left(\Omega ; L^{1}(t, T ; H)\right)$ and $g \in L_{\mathbb{F}}^{2}(t, T ; H)$.

Lemma 3.1 The equation (3.1) admits a unique mild solution $x(\cdot) \in C_{\mathbb{F}}\left([t, T] ; L^{2}(\Omega ; H)\right)$. Moreover,

$$
|x(\cdot)|_{C_{\mathbb{F}}\left([t, T] ; L^{2}(\Omega ; H)\right)} \leq \mathcal{C}\left(|\eta|_{L_{\mathcal{F}_{t}}^{2}(\Omega ; H)}+|f|_{L_{\mathbb{F}}^{2}\left(\Omega ; L^{1}(t, T ; H)\right)}+|g|_{L_{\mathbb{F}}^{2}(t, T ; H)}\right) .
$$

Next, consider the following backward stochastic evolution equation (BSEE for short):

$$
\left\{\begin{array}{l}
d y=-\left[\left(A+A_{1}\right)^{*} y+C^{*} z+h\right] d s+z d W(s) \text { in }[t, T), \\
y(T)=\xi
\end{array}\right.
$$

Here $\xi \in L_{\mathcal{F}_{T}}^{2}(\Omega ; H)$ and $h \in L_{\mathbb{F}}^{2}\left(\Omega ; L^{1}(0, T ; H)\right)$. We have the following result:

Lemma 3.2 The equation (3.3) admits a unique mild solution $(y(\cdot), z(\cdot)) \in L_{\mathbb{F}}^{2}(\Omega ; C([0, T]$; $H)) \times L_{\mathbb{F}}^{2}(0, T ; H)$, and

$$
|(y(\cdot), z(\cdot))|_{L_{\mathbb{F}}^{2}(\Omega ; C([0, T] ; H)) \times L_{\mathbb{F}}^{2}(0, T ; H)} \leq \mathcal{C}\left(|\xi|_{L_{\mathcal{F}_{T}}^{2}(\Omega ; H)}+|h|_{L_{\mathbb{F}}^{2}\left(\Omega ; L^{1}(0, T ; H)\right)}\right) .
$$

Next, we recall the following result.

Lemma 3.3 Let $\bar{\Theta}(\cdot)$ be an optimal feedback operator of Problem (SLQ). Let $\zeta \in H$. The following forward-backward stochastic evolution equation (FBSEE for short) admits a mild solution $(\bar{x}(\cdot), \bar{y}(\cdot), \bar{z}(\cdot)) \in C_{\mathbb{F}}\left([t, T] ; L^{2}(\Omega ; H)\right) \times C_{\mathbb{F}}\left([t, T] ; L^{2}(\Omega ; H)\right) \times L_{\mathbb{F}}^{2}(t, T ; H)$ :

$$
\begin{cases}d x=\left(A+A_{1}+B \bar{\Theta}\right) x d s+(C+D \bar{\Theta}) x d W(s) & \text { in }(t, T], \\ d y=-\left[\left(A+A_{1}\right)^{*} y+C^{*} z+Q x\right] d s+z d W(s) & \text { in }[t, T), \\ x(t)=\zeta, \quad y(T)=G x(T), & \end{cases}
$$

and the following condition holds:

$$
B^{*} \bar{y}+D^{*} \bar{z}+R \Theta \bar{x}=0, \quad \text { a.e. }(s, \omega) \in[t, T] \times \Omega .
$$


If $A_{1}, C \in L^{\infty}(0, T ; \mathcal{L}(H))$ and $B, D \in L^{\infty}(0, T ; \mathcal{L}(U ; H))$, Lemma 3.3 is a trivial corollary of Theorem 5.2 in [24]. The general case can be handled similarly. For the readers' convenience, we give the proof in the appendix.

Consider the following SEE:

$$
\left\{\begin{array}{l}
d \tilde{x}=\left[-A-A_{1}-B \bar{\Theta}+(C+D \bar{\Theta})^{2}\right]^{*} \tilde{x} d s-(C+D \bar{\Theta})^{*} \tilde{x} d W(s) \quad \text { in }(t, T] \\
\tilde{x}(t)=\zeta
\end{array}\right.
$$

where $\zeta \in H$. If $A$ generates a $C_{0}$-group, then $-A^{*}$ also generates a $C_{0}$-group. In this case, by Lemma 3.1 , the equation (3.7) admits a unique mild solution $\tilde{x}(\cdot) \in C_{\mathbb{F}}\left([t, T] ; L^{2}(\Omega ; H)\right)$.

Let $\left\{\varphi_{i}\right\}_{i=1}^{\infty}$ be an orthonormal basis of $U$. For each $n \in \mathbb{N}$, denote by $\Gamma_{n}\left(\right.$ resp. $\left.\widetilde{\Gamma}_{n}\right)$ the projection operator from $H($ resp. $U)$ to $H_{n} \triangleq \operatorname{span}_{1 \leq j \leq n}\left\{e_{j}\right\}\left(\right.$ resp. $\left.U_{n} \triangleq \operatorname{span}_{1 \leq j \leq n}\left\{\varphi_{j}\right\}\right)$. Write

$$
\begin{array}{ll}
A_{n}=\Gamma_{n} A \Gamma_{n}, & A_{1, n}=\Gamma_{n} A_{1} \Gamma_{n}, \quad B_{n}=\Gamma_{n} B \widetilde{\Gamma}_{n}, \quad C_{n}=\Gamma_{n} C \Gamma_{n}, \quad D_{n}=\Gamma_{n} D \widetilde{\Gamma}_{n}, \\
Q_{n}=\Gamma_{n} Q \Gamma_{n}, & R_{n}=\widetilde{\Gamma}_{n} R \widetilde{\Gamma}_{n}, \quad G_{n}=\Gamma_{n} G \Gamma_{n}, \quad \Theta_{n}=\widetilde{\Gamma}_{n} \bar{\Theta} \Gamma_{n} .
\end{array}
$$

Denote by $\left\{\lambda_{j}\right\}_{j=1}^{\infty}$ the eigenvalues of $A$ such that $A e_{j}=\lambda_{j} e_{j}$ (recall that $\left\{e_{j}\right\}_{j=1}^{\infty}$ is the eigenfunctions of $A$ ). Then,

$$
e^{A_{n} s} \zeta=\sum_{j=1}^{n} e^{\lambda_{j} s}\left\langle\zeta, e_{j}\right\rangle e_{j}=e^{A s} \Gamma_{n} \zeta, \quad \forall \zeta \in H
$$

Thus,

$$
\lim _{n \rightarrow+\infty} e^{A_{n} s} \zeta=e^{A s} \zeta, \quad \forall s \in[0, T], \quad \zeta \in H
$$

Similarly, we can get that

$$
\lim _{n \rightarrow+\infty} e^{A_{n}^{*} s} \zeta=e^{A^{*} s} \zeta, \quad \forall s \in[0, T], \quad \zeta \in H
$$

Further, it is easy to show that

$$
\begin{gathered}
\lim _{n \rightarrow+\infty} A_{1, n} \zeta=A_{1} \zeta, \quad \lim _{n \rightarrow+\infty} C_{n} \zeta=C \zeta, \quad \lim _{n \rightarrow+\infty} G_{n} \zeta=G \zeta, \\
\lim _{n \rightarrow+\infty} Q_{n} \zeta=Q \zeta, \quad \lim _{n \rightarrow+\infty} \Theta_{n} \zeta=\bar{\Theta} \zeta, \\
\quad \text { for all } \zeta \in H \text { and a.e. }(s, \omega) \in[0, T] \times \Omega, \\
\lim _{n \rightarrow+\infty} B_{n} \varsigma=B \varsigma, \quad \lim _{n \rightarrow+\infty} D_{n} \varsigma=D \varsigma, \quad \lim _{n \rightarrow+\infty} R_{n} \varsigma=R \varsigma, \\
\quad \text { for all } \varsigma \in U \text { and a.e. }(s, \omega) \in[0, T] \times \Omega .
\end{gathered}
$$

Let $\zeta \in H$, consider the following equations:

$$
\begin{cases}d x_{n}=\left(A_{n}+A_{1, n}+B_{n} \Theta_{n}\right) x_{n} d s+\left(C_{n}+D_{n} \Theta_{n}\right) x_{n} d W(s) & \text { in }[t, T], \\ d y_{n}=-\left[\left(A_{n}+A_{1, n}\right)^{*} y_{n}+C_{n}^{*} z_{n}+Q_{n} x_{n}\right] d s+z_{n} d W(s) & \text { in }[t, T], \\ x_{n}(t)=\Gamma_{n} \zeta, \quad y_{n}(T)=G_{n} x_{n}(T) & \end{cases}
$$


and

$$
\left\{\begin{array}{l}
d \tilde{x}_{n}=\left[-A_{n}-A_{1, n}-B_{n} \Theta_{n}+\left(C_{n}+D_{n} \Theta_{n}\right)^{2}\right]^{*} \tilde{x}_{n} d s-\left(C_{n}+D_{n} \Theta_{n}\right)^{*} \tilde{x}_{n} d W(s) \text { in }(t, T], \\
\tilde{x}_{n}(0)=\Gamma_{n} \zeta .
\end{array}\right.
$$

We have the following result.

Lemma 3.4 Let (AS1) and (AS2) hold. For any $\zeta \in H$, it holds that

$$
\left\{\begin{aligned}
\lim _{n \rightarrow+\infty} x_{n}(\cdot)=x(\cdot) & \text { in } C_{\mathbb{F}}\left([t, T] ; L^{2}(\Omega ; H)\right), \\
\lim _{n \rightarrow+\infty} y_{n}(\cdot)=y(\cdot) & \text { in } L_{\mathbb{F}}^{2}(\Omega ; C([t, T] ; H)), \\
\lim _{n \rightarrow+\infty} z_{n}(\cdot)=z(\cdot) & \text { in } L_{\mathbb{F}}^{2}(t, T ; H), \\
\lim _{n \rightarrow+\infty} \tilde{x}_{n}(\cdot)=\tilde{x}(\cdot) & \text { in } C_{\mathbb{F}}\left([t, T] ; L^{2}(\Omega ; H)\right) .
\end{aligned}\right.
$$

Let $\Theta(\cdot) \in L^{2}(t, T ; \mathcal{L}(H ; U))$. Consider the following operator-valued equation:

$$
\left\{\begin{array}{l}
\dot{P}+P\left(A+A_{1}+B \Theta\right)+\left(A+A_{1}+B \Theta\right)^{*} P \\
+(C+D \Theta)^{*} P(C+D \Theta)+\Theta^{*} R \Theta+Q=0 \quad \text { in }[t, T), \\
P(T)=G
\end{array}\right.
$$

Definition 3.1 We call $P \in C_{\mathcal{S}}([t, T] ; \mathbb{S}(H))$ a mild solution to (3.17) if for any $s \in[t, T]$,

$$
\begin{aligned}
P(s) \eta= & e^{(T-s) A^{*}} G e^{(T-s) A} \eta+\int_{s}^{T} e^{(\tau-s) A^{*}}\left[P\left(A_{1}+B \Theta\right)+\left(A_{1}+B \Theta\right)^{*} P\right. \\
& \left.+(C+D \Theta)^{*} P(C+D \Theta)+\Theta^{*} R \Theta+Q\right] e^{(\tau-s) A} \eta d \tau, \quad \forall \eta \in H .
\end{aligned}
$$

Proposition 3.1 There is a unique mild solution to (3.17). Moreover,

$$
|P|_{C_{\mathcal{S}}([t, T] ; \mathcal{S}(H))} \leq \mathcal{C} e^{\int_{t}^{T}\left(2\left|A_{1}+B \Theta\right|_{\mathcal{L}(H)}+|C+D \Theta|_{\mathcal{L}(H)}^{2}\right) d s}\left[|G|_{\mathcal{L}(H)}+\int_{t}^{T}\left(|\Theta|_{\mathcal{L}(H ; U)}^{2}|R|_{\mathcal{L}(U)}+|Q|_{\mathcal{L}(H)}\right) d s\right]
$$

The following result illustrates the differentiability of $P$.

Proposition 3.2 Let $P$ be a mild solution to (3.17). Then for any $\eta, \zeta \in D(A),\langle P(\cdot) \eta, \zeta\rangle$ is differentiable in $[t, T]$ and

$$
\begin{aligned}
\frac{d}{d s}\langle P \eta, \zeta\rangle= & -\left\langle P \eta,\left(A+A_{1}+B \Theta\right) \zeta\right\rangle-\left\langle P\left(A+A_{1}+B \Theta\right) \eta, \zeta\right\rangle \\
& -\langle P(C+D \Theta) \eta,(C+D \Theta) \zeta\rangle-\langle R \Theta \eta, \Theta \zeta\rangle-\langle Q \eta, \zeta\rangle .
\end{aligned}
$$

Similarly, we have the following result.

Proposition 3.3 Let $P$ be a mild solution to (2.2). Then for any $\eta, \zeta \in D(A),\langle P(\cdot) \eta, \zeta\rangle$ is differentiable in $[t, T]$ and

$$
\begin{aligned}
\frac{d}{d s}\langle P \eta, \zeta\rangle= & -\left\langle P \eta,\left(A+A_{1}\right) \zeta\right\rangle-\left\langle P\left(A+A_{1}\right) \eta, \zeta\right\rangle \\
& -\langle P C \eta, C \zeta\rangle-\langle Q \eta, \zeta\rangle+\left\langle K^{\dagger} L \eta, L \zeta\right\rangle .
\end{aligned}
$$


The result below gives a relation between the cost functional and the Riccati equation (2.2).

Lemma 3.5 Let $P(\cdot) \in C_{\mathcal{S}}([0, T] ; \mathbb{S}(H))$ be the mild solution to (3.17) with $t=0$. Then for any $(t, \eta) \in[0, T) \times H$ and $u(\cdot) \in \mathcal{U}[t, T]$, we have

$$
\mathcal{J}(t, \eta ; \Theta(\cdot) x(\cdot)+u(\cdot))=\langle P(t) \eta, \eta\rangle+\mathbb{E} \int_{t}^{T}[2\langle(L+K \Theta) x, u\rangle+\langle K u, u\rangle] d s .
$$

In the proof of Lemma 3.5, we use a density argument, i.e., approximating the solution $x$ by a $D(A)$-valued process $x_{\lambda}$, doing calculation for $x_{\lambda}$ and letting $\lambda$ tend to $+\infty$ to get the equality for $x$. The main reason is that one may not apply Itô's formula to $|x(t)|_{H}^{2}$ and $x(t)$ may not take values in $D(A)$. This technique will be used several times in the rest of this papers. Except the proof for Lemma 3.5. we omit such process and apply Itô's formula to $|x(t)|_{H}^{2}$ directly and assume that $x(t)$ belongs to $D(A)$. Hence, we give the proof here rather than put it in the appendix.

Proof of Lemma 3.5. For any $(t, \eta) \in[0, T) \times H$ and $u(\cdot) \in \mathcal{U}[t, T]$, let $x(\cdot)$ be the solution to

$$
\left\{\begin{array}{l}
d x=\left[\left(A+A_{1}+B \Theta\right) x+B u\right] d s+[(C+D \Theta) x+D u] d W(s) \quad \text { in }(t, T], \\
x(t)=\eta
\end{array}\right.
$$

Let $\mathbb{R}(\lambda) \triangleq \lambda I(\lambda I-A)^{-1}$ for $\lambda \in \rho(A)$ (the resolvent of $A$ ) and $x_{\lambda}(\cdot)=\mathbb{R}(\lambda) x(\cdot)$. Then $x_{\lambda}(\cdot)$ is the mild solution to

$$
\left\{\begin{array}{l}
d x_{\lambda}=\left\{A x_{\lambda}+\mathbb{R}(\lambda)\left[\left(A_{1}+B \Theta\right) x+B u\right]\right\} d s+\mathbb{R}(\lambda)[(C+D \Theta) x+D u] d W(s) \quad \text { in }(t, T], \\
x_{\lambda}(t)=\mathbb{R}(\lambda) \eta .
\end{array}\right.
$$

By Itô's formula and Proposition 3.2, we have

$$
\begin{aligned}
& \mathbb{E}\left[\left\langle G x_{\lambda}(T), x_{\lambda}(T)\right\rangle+\int_{t}^{T}\left(\left\langle Q x_{\lambda}, x_{\lambda}\right\rangle+\left\langle R\left(\Theta x_{\lambda}+u\right), \Theta x_{\lambda}+u\right\rangle\right) d s\right] \\
= & \langle P(t) \mathbb{R}(\lambda) \eta, \mathbb{R}(\lambda) \eta\rangle+\mathbb{E} \int_{t}^{T}\left\{-\left\langle P(t) x_{\lambda},\left(A+A_{1}+B \Theta\right) x_{\lambda}\right\rangle-\left\langle P(s)\left(A+A_{1}+B \Theta\right) x_{\lambda}, x_{\lambda}\right\rangle\right. \\
& -\left\langle P(C+D \Theta) x_{\lambda},(C+D \Theta) x_{\lambda}\right\rangle-\left\langle R \Theta x_{\lambda}, \Theta x_{\lambda}\right\rangle-\left\langle Q x_{\lambda}, x_{\lambda}\right\rangle+\left\langle P A x_{\lambda}, x_{\lambda}\right\rangle \\
& +\left\langle P \mathbb{R}(\lambda)\left[\left(A_{1}+B \Theta\right) x+B u\right], x_{\lambda}\right\rangle+\left\langle P x_{\lambda}, A x_{\lambda}\right\rangle+\left\langle P x_{\lambda}, \mathbb{R}(\lambda)[(A+B \Theta) x+B u]\right\rangle \\
& +\langle P \mathbb{R}(\lambda)[(C+D \Theta) x+D u], \mathbb{R}(\lambda)[(C+D \Theta) x+D u]\rangle+\left\langle Q x_{\lambda}, x_{\lambda}\right\rangle \\
& \left.+\left\langle R\left(\Theta x_{\lambda}+u\right), \Theta x_{\lambda}+u\right\rangle\right\} d s \\
= & \langle P(t) \eta, \eta\rangle+\mathbb{E} \int_{t}^{T}[2\langle(L+K \Theta) x, u\rangle+\langle K u, u\rangle] d s+F(\lambda),
\end{aligned}
$$

where

$$
F(\lambda)=\langle P(t) \mathbb{R}(\lambda) \eta, \mathbb{R}(\lambda) \eta\rangle-\langle P(t) \eta, \eta\rangle+\mathbb{E} \int_{t}^{T}\left\{\left\langle P \mathbb{R}(\lambda)\left(A_{1}+B \Theta\right) x, x_{\lambda}\right\rangle\right.
$$




$$
\begin{aligned}
& -\left\langle P\left(A_{1}+B \Theta\right) x_{\lambda}, x_{\lambda}\right\rangle+\left\langle P x_{\lambda}, \mathbb{R}(\lambda)(A+B \Theta) x\right\rangle-\left\langle P(s)\left(A_{1}+B \Theta\right) x_{\lambda}, x_{\lambda}\right\rangle \\
& +\langle P \mathbb{R}(\lambda)(C+D \Theta) x, \mathbb{R}(\lambda)(C+D \Theta) x\rangle-\left\langle P(C+D \Theta) x_{\lambda},(C+D \Theta) x_{\lambda}\right\rangle \\
& +\left\langle P \mathbb{R}(\lambda) B u, x_{\lambda}\right\rangle-\langle P B u, x\rangle+\left\langle P x_{\lambda}, \mathbb{R}(\lambda) B u\right\rangle-\langle P x, B u\rangle+\langle P \mathbb{R}(\lambda) D u, \mathbb{R}(\lambda) D u\rangle \\
& -\langle P D u, D u\rangle+\langle P \mathbb{R}(\lambda)(C+D \Theta) x, \mathbb{R}(\lambda) D u\rangle-\langle P(C+D \Theta) x, D u\rangle \\
& +\langle P \mathbb{R}(\lambda) D u, \mathbb{R}(\lambda)(C+D \Theta) x\rangle-\langle P D u,(C+D \Theta) x\rangle\} d s .
\end{aligned}
$$

Noting that for any $\zeta \in H$,

$$
\lim _{\lambda \rightarrow \infty} \mathbb{R}(\lambda) \zeta=\zeta \quad \text { in } H
$$

we have that

$$
\lim _{\lambda \rightarrow \infty}\langle P(t) \mathbb{R}(\lambda) \eta, \mathbb{R}(\lambda) \eta\rangle=\langle P(t) \eta, \eta\rangle
$$

and

$$
\lim _{\lambda \rightarrow \infty} x_{\lambda}=x \quad \text { in } C_{\mathbb{F}}\left([t, T] ; L^{2}(\Omega ; H)\right) .
$$

By (3.23) and (3.24), we get that for a.e. $s \in[t, T]$,

$$
\begin{aligned}
\lim _{\lambda \rightarrow \infty} & {\left[\left\langle P(s) \mathbb{R}(\lambda)\left(A_{1}(s)+B(s) \Theta(s)\right) x(s), x_{\lambda}(s)\right\rangle\right.} \\
& \left.-\left\langle P(s)\left(A_{1}(s)+B(s) \Theta(s)\right) x_{\lambda}(s), x_{\lambda}(s)\right\rangle\right]=0, \quad \mathbb{P} \text {-a.s. }
\end{aligned}
$$

It follows from the definition of $\mathbb{R}(\lambda)$ that

$$
\begin{aligned}
& \left|\left\langle P(s) \mathbb{R}(\lambda)\left(A_{1}(s)+B(s) \Theta(s)\right) x(s), x_{\lambda}(s)\right\rangle-\left\langle P(s)\left(A_{1}(s)+B(s) \Theta(s)\right) x_{\lambda}(s), x_{\lambda}(s)\right\rangle\right| \\
& \leq \mathcal{C}|P(s)|_{\mathcal{L}(H)}\left(\left|A_{1}(s)\right|_{\mathcal{L}(H)}+|B(s)|_{\mathcal{L}(U ; H)}|\Theta(s)|_{\mathcal{L}(H ; U)}\right)|x(s)|_{H}^{2} .
\end{aligned}
$$

This, together with (3.25) and Lebesgue's dominated convergence theorem, implies that

$$
\lim _{\lambda \rightarrow \infty} \mathbb{E} \int_{t}^{T}\left[\left\langle P \mathbb{R}(\lambda)\left(A_{1}+B \Theta\right) x, x_{\lambda}\right\rangle-\left\langle P\left(A_{1}+B \Theta\right) x_{\lambda}, x_{\lambda}\right\rangle\right] d s=0 .
$$

By a similar argument, we can prove that $\lim _{\lambda \rightarrow+\infty} F(\lambda)=0$. Letting $\lambda$ tend to $+\infty$ in both sides of (3.22), we get (3.21).

Remark 3.1 In the derivation of (3.22), we use the fact that the mean value of a stochastic integral of a function quadratically depending on $x$ is zero.

Remark 3.2 Since $x(\cdot)$ may not be $D(A)$-valued, in the proof of Lemma 3.5, we introduce a family of $\left\{x_{\lambda}\right\}_{\lambda \in \rho(A)}$ to apply Proposition 3.2. In the rest of this paper, we omit such procedures to save the space and simply apply Proposition 3.2 to $\langle P(\cdot) x(\cdot), x(\cdot)\rangle$.

Next, we give a result concerning the existence of an optimal control of Problem (SLQ).

Proposition 3.4 Suppose the map $u(\cdot) \mapsto \mathcal{J}(0,0 ; u(\cdot))$ is uniformly convex. Then Problem (SLQ) admits a unique optimal control, and there exists a constant $\alpha \in \mathbb{R}$ such that

$$
V(t, \eta) \geq \alpha|\eta|^{2}, \quad \forall(t, \eta) \in[0, T] \times H
$$


The next result shows that the solution to (3.17) is bounded below.

Proposition 3.5 Let (2.9) hold. Then for any $\Theta(\cdot) \in L^{2}(t, T ; \mathcal{L}(U ; H))$, the solution $P(\cdot) \in$ $C_{\mathcal{S}}([t, T] ; \mathbb{S}(H))$ to (3.17) satisfies

$$
K(s) \geq \lambda I, \quad \text { a.e. } \quad s \in[t, T], \quad \text { and } \quad P(s) \geq \alpha I, \quad \forall s \in[t, T],
$$

where $\alpha \in \mathbb{R}$ is the constant appearing in (3.26).

Let

$$
\widetilde{A}(\cdot) \in L^{1}(0, T ; \mathcal{L}(H)), \quad \widetilde{C}(\cdot) \in L^{2}(0, T ; \mathcal{L}(H)), \quad \widetilde{Q}(\cdot) \in L^{1}(0, T ; \mathbb{S}(H)), \quad \widetilde{G} \in \mathbb{S}(H) .
$$

Consider the following Lyapunov equation:

$$
\left\{\begin{array}{l}
\dot{P}+P(A+\widetilde{A})+(A+\widetilde{A})^{*} P+\widetilde{C}^{*} P \widetilde{C}+\widetilde{Q}=0 \quad \text { in }[t, T), \\
P(T)=\widetilde{G}
\end{array}\right.
$$

Lemma 3.6 The equation (3.28) admits a unique solution $P(\cdot) \in C_{\mathcal{S}}([t, T] ; \mathbb{S}(H))$. Moreover, if

$$
\widetilde{G} \geq 0, \quad \widetilde{Q}(s) \geq 0, \quad \text { a.e. } s \in[t, T],
$$

then $P(\cdot) \in C_{\mathcal{S}}\left([t, T] ; \overline{\mathbb{S}_{+}}(H)\right)$.

Lemma 3.7 For any $u(\cdot) \in \mathcal{U}[t, T]$, let $x$ be the corresponding solution to (1.1) with $\eta=0$. Then for every $\Theta(\cdot) \in L^{2}(t, T ; \mathcal{L}(H ; U))$, there exists a constant $c_{0}>0$ such that

$$
\mathbb{E} \int_{t}^{T}|u(s)-\Theta(s) x(s)|^{2} d s \geq c_{0} \mathbb{E} \int_{t}^{T}|u(s)|^{2} d s, \quad \forall u(\cdot) \in \mathcal{U}[t, T] .
$$

\section{Proof of Theorem 2.1}

This section is devoted to the proof of Theorem 2.1.

Proof of Theorem [2.1. Proof of assertion i). Take any $u(\cdot) \in \mathcal{U}[t, T]$, let $x(\cdot) \equiv$ $x(\cdot ; t, \eta, u(\cdot))$ be the corresponding state process. Then

$$
\begin{aligned}
& \mathcal{J}(t, \eta ; u(\cdot))=\frac{1}{2} \mathbb{E}\left[\langle G x(T), x(T)\rangle+\int_{t}^{T}(\langle Q x, x\rangle+\langle R u, u\rangle) d s\right] \\
& =\frac{1}{2} \mathbb{E}\langle P(t) \eta, \eta\rangle+\frac{1}{2} \mathbb{E} \int_{t}^{T}\left[\left\langle\left(-P\left(A+A_{1}\right)-\left(A+A_{1}\right)^{*} P-C^{*} P C-Q+L^{*} K^{\dagger} L\right) x, x\right\rangle\right. \\
& \quad+\left\langle P\left(\left(A+A_{1}\right) x+B u\right), x\right\rangle+\left\langle P x,\left(A+A_{1}\right) x+B u\right\rangle+\langle P(C x+D u), C x+D u\rangle \\
& \quad+\langle Q x, x\rangle+\langle R u, u\rangle] d s \\
& =\frac{1}{2} \mathbb{E}\left[\langle P(t) \eta, \eta\rangle+\int_{t}^{T}\left(\left\langle L^{*} K^{\dagger} L x, x\right\rangle+2\langle L x, u\rangle+\langle K u, u\rangle\right) d s\right] .
\end{aligned}
$$


Noting that $L=B^{*} P+D^{*} P C=-K \Theta$, it holds that

$$
\begin{aligned}
& \mathcal{J}(t, \eta ; u(\cdot)) \\
= & \frac{1}{2} \mathbb{E}\left[\langle P(t) \eta, \eta\rangle+\int_{t}^{T}\left(\left\langle L^{*} K^{\dagger} L x, x\right\rangle+2\langle L x, u\rangle+\langle K u, u\rangle\right) d s\right] \\
= & \frac{1}{2} \mathbb{E}\left[\langle P(t) \eta, \eta\rangle+\int_{t}^{T}\left(\left\langle\Theta^{*} K K^{\dagger} K \Theta x, x\right\rangle-2\langle K \Theta x, u\rangle+\langle K u, u\rangle\right) d s\right] \\
= & \frac{1}{2} \mathbb{E}\left[\langle P(t) \eta, \eta\rangle+\int_{t}^{T}(\langle K \Theta x, \Theta x\rangle-2\langle K \Theta x, u\rangle+\langle K u, u\rangle) d s\right] \\
= & \frac{1}{2} \mathbb{E}\left[\langle P(t) \eta, \eta\rangle+\int_{t}^{T}\langle K(u-\Theta x), u-\Theta x\rangle d s\right] \\
= & \mathcal{J}(t, \eta ; \Theta(\cdot) x(\cdot))+\frac{1}{2} \mathbb{E} \int_{t}^{T}\langle K(u-\Theta x), u-\Theta x\rangle d s .
\end{aligned}
$$

Hence,

$$
\mathcal{J}(t, \eta ; \Theta(\cdot) x(\cdot)) \leq \mathcal{J}(t, \eta ; u), \quad \forall u(\cdot) \in \mathcal{U}[t, T]
$$

if and only if $P$ is a regular solution to the Riccati equation (2.2).

Proof of assertion ii). Without loss of generality, we assume that $t=0$. The proof is divided into five steps.

Step 1. In this step, we introduce some operators and their finite dimensional approximation.

Let $\bar{\Theta}(\cdot)$ be an optimal feedback operator of Problem (SLQ) over [0,T]. For each $s \in$ $[0, T]$, define three operators $X_{s}, Y_{s}$ and $\widetilde{X}_{s}$ on $H$ as follows:

$$
X_{s} \zeta=\bar{x}(s ; \zeta), \quad Y_{s} \zeta=\bar{y}(s ; \zeta), \quad \widetilde{X}_{s} \zeta=\tilde{x}(s ; \zeta), \quad \forall \zeta \in H
$$

For a.e. $s \in[0, T]$, define an operator $Z_{s}$ on $H$ by

$$
Z_{s} \zeta=\bar{z}(s ; \zeta), \quad \forall \zeta \in H .
$$

Here $(\bar{x}, \bar{y}, \bar{z})$ solves (3.5) and $\tilde{x}$ solves (3.7). Now we are going to show some properties of the above four operators.

Denote by $I_{n}$ the identity matrix on $\mathbb{R}^{n}$ (or, the identity map on $H_{n}$ ). Consider the following equations:

$$
\begin{cases}d X_{n}=\left(A_{n}+A_{1, n}+B_{n} \Theta_{n}\right) X_{n} d s+\left(C_{n}+D_{n} \Theta_{n}\right) X_{n} d W(s) & \text { in }[0, T], \\ d Y_{n}=-\left[\left(A_{n}+A_{1, n}\right)^{*} Y_{n}+C_{n}^{*} Z_{n}+Q_{n} X_{n}\right] d t+Z_{n} d W(t) & \text { in }[0, T], \\ X_{n}(0)=I_{n}, \quad Y_{n}(T)=G_{n} X_{n}(T) & \end{cases}
$$

and

$$
\left\{\begin{array}{rlr}
d \widetilde{X}_{n}= & {\left[-A_{n}-A_{1, n}-B_{n} \Theta_{n}+\left(C_{n}+D_{n} \Theta_{n}\right)\left(C_{n}+D_{n} \Theta_{n}\right)^{\top}\right]^{\top} \widetilde{X}_{n} d s} \\
& -\left(C_{n}+D_{n} \Theta_{n}\right)^{\top} \widetilde{X}_{n} d W(s) & \text { in }[0, T], \\
\widetilde{X}_{n}(0)= & I_{n} . &
\end{array}\right.
$$


Clearly, both (4.3) and (4.4) can be viewed as $\mathbb{R}^{n \times n} \equiv \mathbb{R}^{n^{2}}$-valued equations. They admit unique solutions $\left(X_{n}, Y_{n}, Z_{n}\right) \in C_{\mathbb{F}}\left([0, T] ; L^{2}\left(\Omega ; \mathbb{R}^{n \times n}\right)\right) \times C_{\mathbb{F}}\left([0, T] ; L^{2}\left(\Omega ; \mathbb{R}^{n \times n}\right)\right) \times$ $L_{\mathbb{F}}^{2}\left(0, T ; \mathbb{R}^{n \times n}\right)$ and $\widetilde{X}_{n} \in C_{\mathbb{F}}\left([0, T] ; L^{2}\left(\Omega ; \mathbb{R}^{n \times n}\right)\right)$, respectively. By Itô's formula, we see that

$$
\begin{aligned}
& X_{n}(s) \tilde{X}_{n}(s)^{\top}-X_{n}(0) \tilde{X}_{n}(0)^{\top} \\
= & \int_{0}^{s}\left\{\left\langle\left(A_{n}+A_{1, n}+B_{n} \Theta_{n}\right) X_{n}, \tilde{X}^{\top}\right\rangle+\left\langle X_{n}, \tilde{X}_{n}^{\top}\left[-A_{n}-A_{1, n}-B_{n} \Theta_{n}+\left(C_{n}+D_{n} \Theta_{n}\right)^{2}\right]\right\rangle\right. \\
& +\left\langle\left(C_{n}+D_{n} \Theta_{n}\right) X_{n},-\tilde{X}_{n}^{\top}\left(C_{n}+D_{n} \Theta_{n}\right)\right\rangle d r \\
& +\int_{0}^{s}\left[\left\langle\left(C_{n}+D_{n} \Theta_{n}\right) X_{n}, \tilde{X}_{n}^{\top}\right\rangle+\left\langle X_{n},-\tilde{X}_{n}^{\top}\left(C_{n}+D_{n} \Theta_{n}\right)\right\rangle\right] d W(r)=0 .
\end{aligned}
$$

Consequently,

$$
\widetilde{X}(s)_{n}^{\top}=X_{n}(s)^{-1}, \quad \mathbb{P} \text {-a.s. }, \quad \forall s \in[0, T] .
$$

Let $\zeta \in H$. It is an easy matter to see that $x_{n}(s)=X_{n}(s) \Gamma_{n} \zeta, y_{n}(s)=Y_{n}(s) \Gamma_{n} \zeta$, $z_{n}(s)=Z_{n}(s) \Gamma_{n} \zeta$ and $\tilde{x}_{n}(s)=\tilde{X}_{n}(s) \Gamma_{n} \zeta$. Thus, $\left(X_{n}(\cdot) \Gamma_{n} \zeta, Y_{n}(\cdot) \Gamma_{n} \zeta, Z_{n}(\cdot) \Gamma_{n} \zeta\right)$ solves (3.14) and $\widetilde{X}_{n}(\cdot) \Gamma_{n} \zeta$ solves (3.15). For each $s \in[0, T]$, define three operators $X_{n, s}, Y_{n, s}$ and $\widetilde{X}_{n, s}$ on $H$ as follows:

$$
X_{n, s} \Gamma_{n} \zeta=X_{n}(s) \Gamma_{n} \zeta, \quad Y_{n, s} \Gamma_{n} \zeta=Y_{n}(s) \Gamma_{n} \zeta, \quad \widetilde{X}_{n, s} \Gamma_{n} \zeta=\tilde{X}_{n}(s) \Gamma_{n} \zeta, \quad \forall \zeta \in H
$$

For a.e. $s \in[0, T]$, define an operator $Z_{n, s}$ on $H_{n}$ by

$$
Z_{n, s} \Gamma_{n} \zeta=Z_{n}(s) \Gamma_{n} \zeta, \quad \forall \zeta \in H
$$

In view of (4.6), (4.7) and (3.16), we find that

$$
\left\{\begin{array}{cl}
\lim _{n \rightarrow+\infty} X_{n, s} \Gamma_{n} \zeta=X_{s} \zeta & \text { strongly in } L_{\mathcal{F}_{s}}^{2}(\Omega ; H), \\
\lim _{n \rightarrow+\infty} Y_{n, s} \Gamma_{n} \zeta=Y_{s} \zeta & \text { strongly in } L_{\mathcal{F}_{s}}^{2}(\Omega ; H), \\
\lim _{n \rightarrow+\infty} Z_{n, s} \Gamma_{n} \zeta=Z_{s} \zeta & \text { strongly in } L_{\mathbb{F}}^{2}(0, T ; H), \\
\lim _{n \rightarrow+\infty} \widetilde{X}_{n, s} \Gamma_{n} \zeta=\widetilde{X}_{s} \zeta & \text { strongly in } L_{\mathcal{F}_{s}}^{2}(\Omega ; H) .
\end{array}\right.
$$

Step 2. In this step, we give an explicit formula of $P(\cdot)$.

By the well-posedness results for the equations (3.14) and (3.15), and the fact that both $A$ and $-A^{*}$ generate $C_{0}$-semigroups on $H$ (because $A$ generates a $C_{0}$-group on $H$ ), we see that

$$
\begin{aligned}
& \left|X_{n, s} \Gamma_{n} \zeta\right|_{L_{\mathcal{F}_{s}}^{2}(\Omega ; H)} \leq \mathcal{C}|\zeta|_{H}, \quad\left|Y_{n, s} \Gamma_{n} \zeta\right|_{L_{\mathcal{F}_{s}}^{2}(\Omega ; H)} \leq \mathcal{C}|\zeta|_{H}, \\
& \left|Z_{n,} \Gamma_{n} \zeta\right|_{L_{\mathbb{F}}^{2}(0, T ; H)} \leq \mathcal{C}|\zeta|_{H}, \quad\left|\widetilde{X}_{n, s} \Gamma_{n} \zeta\right|_{L_{\mathcal{F}_{t}}^{2}(\Omega ; H)} \leq \mathcal{C}|\zeta|_{H},
\end{aligned}
$$

where the constant $\mathcal{C}$ is independent of $n$. This implies that

$$
\begin{aligned}
\left|X_{n, s} \Gamma_{n}\right|_{\mathcal{L}\left(H ; L_{\mathcal{F}_{s}}^{2}(\Omega ; H)\right)} \leq \mathcal{C}, \quad\left|Y_{n, s} \Gamma_{n}\right|_{\mathcal{L}\left(H ; L_{\mathcal{F}_{s}}^{2}(\Omega ; H)\right)} \leq \mathcal{C}, \\
\left|Z_{n,} \Gamma_{n}\right|_{\mathcal{L}\left(H ; L_{\mathbb{F}}^{2}(0, T ; H)\right)} \leq \mathcal{C}, \quad\left|\widetilde{X}_{n, s} \Gamma_{n}\right|_{\mathcal{L}\left(H ; L_{\mathcal{F}_{s}}^{2}(\Omega ; H)\right)} \leq \mathcal{C}
\end{aligned}
$$


By (4.9) and using [23, Theorems 5.2-5.3], we deduce that, there exist subsequences $\left\{X_{n_{k}, s}\right\}_{k=1}^{\infty} \subset\left\{X_{n, s}\right\}_{n=1}^{\infty},\left\{Y_{n_{k}, s}\right\}_{k=1}^{\infty} \subset\left\{Y_{n, s}\right\}_{n=1}^{\infty},\left\{Z_{n_{k}, s}\right\}_{k=1}^{\infty} \subset\left\{Z_{n, s}\right\}_{n=1}^{\infty}$ and $\left\{\tilde{X}_{n_{k}, s}\right\}_{k=1}^{\infty} \subset$ $\left\{\widetilde{X}_{n, s}\right\}_{n=1}^{\infty}$ (these sequences may depend on $s$ ), and (pointwise defined) operators $X_{1}(s, \cdot)$, $Y_{1}(s, \cdot), \widetilde{X}_{1}(s, \cdot) \in \mathcal{L}\left(H ; L_{\mathcal{F}_{s}}^{2}(\Omega ; H)\right)($ for each $s \in[0, T])$ and $Z_{1}(\cdot, \cdot) \in \mathcal{L}\left(H ; L_{\mathbb{F}}^{2}(0, T ; H)\right)$ such that

$$
\left\{\begin{array}{cl}
\lim _{k \rightarrow+\infty} X_{n_{k}, s} \Gamma_{n_{k}} \zeta=X_{1}(s, \cdot) \zeta & \text { weakly in } L_{\mathcal{F}_{s}}^{2}(\Omega ; H), \\
\lim _{k \rightarrow+\infty} Y_{n_{k}, s} \Gamma_{n_{k}} \zeta=Y_{1}(t, \cdot) \zeta & \text { weakly in } L_{\mathcal{F}_{s}}^{2}(\Omega ; H), \\
\lim _{k \rightarrow+\infty} Z_{n_{k}, s} \Gamma_{n_{k}} \zeta=Z_{1}(\cdot, \cdot) \zeta & \text { weakly in } L_{\mathbb{F}}^{2}(0, T ; H), \\
\lim _{k \rightarrow+\infty} \widetilde{X}_{n_{k}, s} \Gamma_{n_{k}} \zeta=\widetilde{X}_{1}(s, \cdot) \zeta & \text { weakly in } L_{\mathcal{F}_{s}}^{2}(\Omega ; H),
\end{array}\right.
$$

and that

$$
\begin{aligned}
& \left|X_{1}(s, \cdot) \zeta\right|_{L_{\mathcal{F}_{S}}^{2}(\Omega ; H)} \leq \mathcal{C}|\zeta|_{H}, \quad\left|Y_{1}(s, \cdot) \zeta\right|_{L_{\mathcal{F}_{\mathcal{S}}}^{2}(\Omega ; H)} \leq \mathcal{C}|\zeta|_{H} \\
& \left|Z_{1}(\cdot, \cdot) \zeta\right|_{L_{\mathbb{F}}^{2}(0, T ; H)} \leq \mathcal{C}|\zeta|_{H}, \quad\left|\widetilde{X}_{1}(s, \cdot) \zeta\right|_{L_{\mathcal{F}_{\mathcal{S}}}^{2}(\Omega ; H)} \leq \mathcal{C}|\zeta|_{H}
\end{aligned}
$$

It follows from (4.8) and (4.10) that

$$
\begin{aligned}
& X(s, \cdot) \triangleq X_{s}(\cdot)=X_{1}(s, \cdot), \quad Y(s, \cdot) \triangleq Y_{s}(\cdot)=Y_{1}(s, \cdot), \\
& Z(s, \cdot) \triangleq Z_{s}(\cdot)=Z_{1}(s, \cdot), \quad \widetilde{X}(t, \cdot) \triangleq \widetilde{X}_{s}(\cdot)=\widetilde{X}_{1}(s, \cdot) .
\end{aligned}
$$

By (3.6), (4.1), (4.2) and (4.12), we find that

$$
R \Theta X+B^{*} Y+D^{*} Z=0, \quad \text { a.e. }(s, \omega) \in[0, T] \times \Omega .
$$

From (4.5), (4.8), (4.10) and (4.12), it is easy to see that for any $s \in[0, T], X(s) \widetilde{X}(s)^{*}=I$, $\mathbb{P}$-a.s., that is, for any $s \in[0, T], \widetilde{X}(s)^{*}=X(s)^{-1}, \mathbb{P}$-a.s.

Put

$$
P(\cdot)=Y(\cdot) X(\cdot)^{-1}, \quad \Pi(\cdot)=Z(\cdot) X(\cdot)^{-1} .
$$

It follows from (4.13) that

$$
B^{*} P+D^{*} \Pi+R \Theta=0, \quad \text { a.e. }(s, \omega) \in[0, T] \times \Omega .
$$

Step 3. In this step, we give an estimate of the norm of $P(\cdot)$ introduced in Step 2. Let $s \in[0, T)$ and $\eta \in L_{\mathcal{F}_{s}}^{2}(\Omega ; H)$. Consider the following FBSEE:

$$
\begin{cases}d x^{s}(r)=\left(A+A_{1}+B \Theta\right) x^{s} d r+(C+D \Theta) x^{s} d W(r) & \text { in }(s, T] \\ d y^{s}(r)=-\left[\left(A+A_{1}\right)^{*} y^{s}+C^{*} z^{s}+Q x^{s}\right] d r+z^{s} d W(r) & \text { in }[s, T), \\ x^{s}(s)=\eta, \quad y^{s}(T)=G x^{s}(T) & \end{cases}
$$

By Lemma 3.3, it is easy to see that (4.16) admits a unique solution $\left(x^{s}(\cdot), y^{s}(\cdot), z^{s}(\cdot)\right)(\equiv$ $\left.\left(x^{s}(\cdot ; \eta), y^{s}(\cdot ; \eta), z^{s}(\cdot ; \eta)\right)\right) \in C_{\mathbb{F}}\left([s, T] ; L^{2}(\Omega ; H)\right) \times C_{\mathbb{F}}\left([s, T] ; L^{2}(\Omega ; H)\right) \times L_{\mathbb{F}}^{2}(s, T ; H)$ such that

$$
R \Theta x^{s}(r)+B^{*} y^{s}(r)+D^{*} z^{s}(r)=0, \quad \text { a.e. }(r, \omega) \in(s, T) \times \Omega .
$$


For every $r \in[s, T]$, define two families of operators $X_{r}^{s}$ and $Y_{r}^{s}$ on $L_{\mathcal{F}_{s}}^{2}(\Omega ; H)$ :

$$
X_{r}^{s} \eta \triangleq x^{s}(r ; \eta), \quad Y_{r}^{s} \eta \triangleq y^{s}(r ; \eta) .
$$

It follows from Lemmas 3.1 and 3.2 that for all $r \in[s, T]$ and $\eta \in L_{\mathcal{F}_{s}}^{2}(\Omega ; H)$,

$$
\left|X_{r}^{s} \eta\right|_{L_{\mathcal{F}_{r}}^{2}(\Omega ; H)} \leq \mathcal{C}|\eta|_{L_{\mathcal{F}_{s}}^{2}(\Omega ; H)}, \quad\left|Y_{r}^{s} \eta\right|_{L_{\mathcal{F}_{r}}^{2}(\Omega ; H)} \leq \mathcal{C}|\eta|_{L_{\mathcal{F}_{s}}^{2}(\Omega ; H)}
$$

This indicates that $X_{r}^{s}$ and $Y_{r}^{s}$ belong to $\mathcal{L}\left(L_{\mathcal{F}_{s}}^{2}(\Omega ; H) ; L_{\mathcal{F}_{r}}^{2}(\Omega ; H)\right)$ for every $r \in[s, T]$.

By (4.16), it is easy to see that, for any $\zeta \in H$,

$$
X_{r}^{s} X(s) \zeta=x^{s}(r ; X(s) \zeta)=x(r ; \zeta)
$$

Thus,

$$
Y_{s}^{s} X(s) \zeta=y^{s}(s ; X(s) \zeta)=Y(s) \zeta
$$

This implies that

$$
Y_{s}^{s}=Y(s) \widetilde{X}(s)^{*} \quad \text { for all } s \in[0, T], \quad \mathbb{P} \text {-a.s. }
$$

Let $\eta, \xi \in L_{\mathcal{F}_{s}}^{2}(\Omega ; H)$. Since $Y_{r}^{s} \eta=y^{s}(r ; \eta)$ and $X_{r}^{s} \xi=x^{s}(r ; \xi)$, applying Itô's formula to $\left\langle y^{s}(\cdot ; \eta), x^{s}(\cdot ; \xi)\right\rangle$ and noting (4.16)-(4.17), we obtain that

$$
\mathbb{E}\left\langle G X_{T}^{s} \eta, X_{T}^{s} \xi\right\rangle-\mathbb{E}\left\langle Y_{s}^{s} \eta, \xi\right\rangle=-\mathbb{E} \int_{s}^{T}\left(\left\langle Q X_{r}^{s} \eta, X_{r}^{s} \xi\right\rangle+\left\langle R \Theta X_{r}^{s} \eta, \Theta X_{r}^{s} \xi\right\rangle\right) d r
$$

Therefore,

$$
\mathbb{E}\left\langle Y_{s}^{s} \eta, \xi\right\rangle=\mathbb{E}\left\langle\left(X_{T}^{s}\right)^{*} G X_{T}^{s} \eta+\mathbb{E} \int_{s}^{T}\left(\left(X_{r}^{s}\right)^{*} Q X_{r}^{s} \eta+\left(X_{r}^{s}\right)^{*} \Theta^{*} R \Theta X_{r}^{s} \eta\right) d r, \xi\right\rangle .
$$

We conclude from this that, for any $\eta \in L_{\mathcal{F}_{s}}^{2}(\Omega ; H)$,

$$
Y_{s}^{s} \eta=\mathbb{E}\left(\left(X_{T}^{s}\right)^{*} G X_{T}^{s} \eta+\mathbb{E} \int_{s}^{T}\left(\left(X_{r}^{s}\right)^{*} Q X_{r}^{s} \eta+\left(X_{r}^{s}\right)^{*} \Theta^{*} R \Theta X_{r}^{s} \eta\right) d r \mid \mathcal{F}_{s}\right) .
$$

It deduces that $Y(s) \tilde{X}(s)^{*}=Y_{s}^{s}$ is symmetric for any $s \in[0, T], \mathbb{P}$-a.s. Further, (4.20) together with (4.18) implies that for any $s \in[0, T]$ and $\eta \in L_{\mathcal{F}_{s}}^{2}(\Omega ; H)$,

$$
\mathbb{E}\left|Y_{s}^{s} \eta\right|_{H}^{2} \leq \mathcal{C} \mathbb{E}|\eta|_{H}^{2}
$$

where $\mathcal{C}$ is independent of $s \in[0, T)$. According to (4.21), we find that

$$
\left|Y(s) \tilde{X}(s)^{*}\right|_{\mathcal{L}\left(L_{\mathcal{F}_{s}}^{2}(\Omega ; H) ; L_{\mathcal{F}_{s}}^{2}(\Omega ; H)\right)} \leq \mathcal{C}
$$

It follows from (4.14), (4.19) and (4.22) that, for some positive constant $\mathcal{C}$,

$$
|P(s)|_{\mathcal{L}\left(L_{\mathcal{F}_{s}}^{2}(\Omega ; H) ; L_{\mathcal{F}_{s}}^{2}(\Omega ; H)\right)} \leq \mathcal{C}, \quad \forall s \in[0, T] .
$$

Step 4. In this step, we show that $P(\cdot)$ is a mild solution to an operator-valued differential equation. 
Let $s \in[0, T)$ and $\zeta \in L_{\mathcal{F}_{s}}^{2}(\Omega ; H)$. Consider the following FBSEE:

$$
\begin{cases}d x_{n}^{s}(\tau)=\left(A_{n}+A_{1, n}+B_{n} \Theta_{n}\right) x_{n}^{s} d \tau+\left(C_{n}+D_{n} \Theta_{n}\right) x_{n}^{s} d W(\tau) & \text { in }[s, T] \\ d y_{n}^{s}(\tau)=-\left[\left(A_{n}+A_{1, n}\right)^{*} y_{n}^{s}+C_{n}^{*} z_{n}^{s}+Q_{n} x_{n}^{s}\right] d \tau+z_{n}^{s} d W(\tau) & \text { in }[s, T] \\ x_{n}^{s}(s)=\zeta, \quad y_{n}^{s}(T)=G_{n} x_{n}^{s}(T) . & \end{cases}
$$

For every $r \in[s, T]$, define two families of operators $X_{r}^{n, s}$ and $Y_{r}^{n, s}$ on $L_{\mathcal{F}_{s}}^{2}(\Omega ; H)$ as follows:

$$
X_{r}^{n, s} \zeta \triangleq x_{n}^{s}(r ; \zeta), \quad Y_{r}^{n, s} \zeta \triangleq y_{n}^{s}(r ; \zeta)
$$

Similar to the proofs of (4.8) and (4.19), we can show that

$$
\begin{cases}\lim _{n \rightarrow+\infty} X_{r}^{n, s} \zeta=X_{r}^{s} \zeta & \text { in } L_{\mathcal{F}_{r}}^{2}(\Omega ; H) \\ \lim _{n \rightarrow+\infty} Y_{r}^{n, s} \zeta=Y_{r}^{s} \zeta & \text { in } L_{\mathcal{F}_{r}}^{2}(\Omega ; H)\end{cases}
$$

and

$$
Y_{s}^{n, s}=Y_{n}(s) \widetilde{X}_{n}(s)^{\top} \quad \text { for all } s \in[0, T], \quad \mathbb{P} \text {-a.s. }
$$

Let $P_{n}(\cdot)=Y_{n}(\cdot) \widetilde{X}_{n}(\cdot)^{\top}$ and $\Pi_{n}(\cdot)=Z_{n}(\cdot) \widetilde{X}_{n}(\cdot)^{\top}$. We conclude from (4.19), (4.25) and (4.26) that

$$
\lim _{n \rightarrow+\infty} P_{n}(s) \Gamma_{n} \eta=P(s) \eta \quad \text { in } H, \quad \forall \eta \in H
$$

By Itô's formula,

$$
\begin{aligned}
d P_{n}= & \left\{-\left[\left(A_{n}+A_{1, n}\right)^{\top} Y_{n}+C_{n}^{\top} Z_{n}+Q_{n}^{\top} X_{n}^{\top}\right] \tilde{X}_{n}^{\top}+Y_{n} \tilde{X}_{n}^{\top}\left[\left(C_{n}+D_{n} \Theta_{n}\right)^{2}-A_{n}-B_{n} \Theta_{n}\right]\right. \\
& \left.-Z_{n} \tilde{X}_{n}^{\top}\left(C_{n}+D_{n} \Theta_{n}\right)\right\} d \tau+\left[Z_{n} \tilde{X}_{n}^{\top}-Y_{n} \tilde{X}_{n}^{\top}\left(C_{n}+D_{n} \Theta_{n}\right)\right] d W(\tau) \\
= & \left\{-\left(A_{n}+A_{1, n}\right)^{\top} P_{n}-C_{n}^{\top} \Pi_{n}-Q_{n}+P_{n}\left[\left(C_{n}+D_{n} \Theta_{n}\right)^{2}-\left(A_{n}+A_{1, n}\right)-B_{n} \Theta_{n}\right]\right. \\
& \left.-\Pi_{n}\left(C_{n}+D_{n} \Theta_{n}\right)\right\} d \tau+\left[\Pi_{n}-P_{n}\left(C_{n}+D_{n} \Theta_{n}\right)\right] d W(\tau) .
\end{aligned}
$$

Let $\Lambda_{n}=\Pi_{n}-P_{n}\left(C_{n}+D_{n} \Theta_{n}\right)$. Then

$$
\begin{aligned}
d P_{n}= & \left\{-\left(A_{n}+A_{1, n}\right)^{\top} P_{n}-C_{n}^{\top}\left[\Lambda_{n}+P_{n}\left(C_{n}+D_{n} \Theta_{n}\right)\right]-Q_{n}+P_{n}\left[\left(C_{n}+D_{n} \Theta\right)^{2}\right.\right. \\
& \left.\left.-\left(A_{n}+A_{1, n}\right)-B_{n} \Theta_{n}\right]-\left[\Lambda_{n}+P_{n}\left(C_{n}+D_{n} \Theta_{n}\right)\right]\left(C_{n}+D_{n} \Theta_{n}\right)\right\} d \tau+\Lambda_{n} d W(\tau) \\
= & {\left[-P_{n}\left(A_{n}+A_{1, n}\right)-\left(A_{n}+A_{1, n}\right)^{\top} P_{n}-\Lambda_{n} C_{n}-C_{n}^{\top} \Lambda_{n}-C_{n}^{\top} P_{n} C_{n}\right.} \\
& \left.-\left(P_{n} B_{n}+C_{n}^{\top} P_{n} D_{n}+\Lambda_{n} D_{n}\right) \Theta_{n}-Q_{n}\right] d \tau+\Lambda_{n} d W(\tau),
\end{aligned}
$$

and $P_{n}(T)=G_{n}$. This implies that $\left(P_{n}(\cdot), \Lambda_{n}(\cdot)\right)$ is the adapted solution to (4.28) with deterministic coefficients and final datum. Thus, $P_{n}(\cdot)$ is deterministic and $\Lambda_{n}(\cdot)=0$. Then, (4.28) becomes

$$
\dot{P}_{n}+P_{n}\left(A_{n}+A_{1, n}\right)+\left(A_{n}+A_{1, n}\right)^{\top} P_{n}+C_{n}^{\top} P_{n} C_{n}+\left(P_{n} B_{n}+C_{n}^{\top} P_{n} D_{n}\right) \Theta_{n}+Q_{n}=0 .
$$


Thus,

$$
\begin{aligned}
P_{n}(s)=e^{A_{n}^{\top}(T-s)} G_{n} e^{A_{n}(T-s)}+\int_{s}^{T} e^{A_{n}^{\top}(r-s)} & {\left[P_{n} A_{1, n}+A_{1, n}^{\top} P_{n}+C_{n}^{\top} P_{n} C_{n}\right.} \\
& \left.+\left(P_{n} B_{n}+C_{n}^{\top} P_{n} D_{n}\right) \Theta_{n}+Q_{n}\right] e^{A_{n}(r-s)} d r .
\end{aligned}
$$

Therefore, for any $\zeta \in H$,

$$
\begin{aligned}
P_{n}(s) \Gamma_{n} \zeta=e^{A_{n}^{\top}(T-s)} G_{n} e^{A_{n}(T-s)} \Gamma_{n} \eta+ & \int_{s}^{T} e^{A_{n}^{\top}(r-s)}\left[P_{n} A_{1, n}+A_{1, n}^{\top} P_{n}+C_{n}^{\top} P_{n} C_{n}\right. \\
& \left.+\left(P_{n} B_{n}+C_{n}^{\top} P_{n} D_{n}\right) \Theta_{n}+Q_{n}\right] e^{A_{n}(r-s)} \Gamma_{n} \zeta d r .
\end{aligned}
$$

From (3.8) and (4.27), we know that for any $\zeta \in H$,

$$
\lim _{n \rightarrow \infty} e^{A_{n}^{\top}(T-s)} G_{n} e^{A_{n}(T-s)} \Gamma_{n} \zeta=e^{A^{*}(T-s)} G e^{A(T-s)} \zeta
$$

and

$$
\begin{aligned}
& \lim _{n \rightarrow \infty} e^{A_{n}^{\top}(r-s)}\left[P_{n} A_{1, n}+A_{1, n}^{\top} P_{n}+C_{n}^{\top} P_{n} C_{n}+\left(P_{n} B_{n}+C_{n}^{\top} P_{n} D_{n}\right) \Theta_{n}+Q_{n}\right] e^{A_{n}(r-s)} \Gamma_{n} \zeta \\
& =e^{A^{*}(r-s)}\left[P A_{1}+A_{1}^{*} P+C^{\top} P C+\left(P B+C^{\top} P D\right) \Theta+Q\right] e^{A(r-s)} \zeta .
\end{aligned}
$$

By (3.8) again, we have that

$$
\begin{aligned}
& \left|e^{A_{n}^{\top}(r-s)}\left[P_{n} A_{1, n}+A_{1, n}^{\top} P_{n}+C_{n}^{\top} P_{n} C_{n}+\left(P_{n} B_{n}+C_{n}^{\top} P_{n} D_{n}\right) \Theta_{n}+Q_{n}\right] e^{A_{n}(r-s)} \Gamma_{n} \zeta\right|_{H} \\
& \leq \mathcal{C}\left|e^{A_{n}^{\top}(r-s)}\right|_{\mathcal{L}(H)}\left[2\left|A_{1, n}^{\top}\right|_{\mathcal{L}(H)}\left|P_{n}\right|_{\mathcal{L}(H)}+\left|C_{n}^{\top}\right|_{\mathcal{L}(H)}\left|P_{n}\right|_{\mathcal{L}(H)}\left|C_{n}\right|_{\mathcal{L}(H)}+\left(\left|P_{n}\right|_{\mathcal{L}(H)}\left|B_{n}\right|_{\mathcal{L}(U ; H)}\right.\right. \\
& \left.\left.\quad+\left|C_{n}^{\top}\right|_{\mathcal{L}(H)}\left|P_{n}\right|_{\mathcal{L}(H)}\left|D_{n}\right|_{\mathcal{L}(U ; H)}\right)\left|\Theta_{n}\right|_{\mathcal{L}(H ; U)}+\left|Q_{n}\right|_{\mathcal{L}(H)}\right]\left|e^{A_{n}(r-s)}\right|_{\mathcal{L}(H)}\left|\Gamma_{n} \zeta\right|_{H} \\
& \leq \mathcal{C}\left|e^{A^{*}(r-s)}\right|_{\mathcal{L}(H)}\left[2\left|A_{1}^{*}\right|_{\mathcal{L}(H)}|P|_{\mathcal{L}(H)}+\left|C^{*}\right|_{\mathcal{L}(H)}|P|_{\mathcal{L}(H)}|C|_{\mathcal{L}(H)}+\left(|P|_{\mathcal{L}(H)}|B|_{\mathcal{L}(U ; H)}\right.\right. \\
& \left.\left.\quad+\left|C^{*}\right|_{\mathcal{L}(H)}|P|_{\mathcal{L}(H)}|D|_{\mathcal{L}(U ; H)}\right)|\Theta|_{\mathcal{L}(H ; U)}+|Q|_{\mathcal{L}(H)}\right]\left|e^{A(r-s)}\right|_{\mathcal{L}(H)}|\zeta|_{H} .
\end{aligned}
$$

This, together with (4.32) and Lebesgue's dominated convergence theorem, implies that

$$
\begin{aligned}
& \lim _{n \rightarrow \infty} \int_{s}^{T} e^{A_{n}^{\top}(r-s)}\left[P_{n} A_{1, n}+A_{1, n}^{\top} P_{n}+C_{n}^{\top} P_{n} C_{n}+\left(P_{n} B_{n}+C_{n}^{\top} P_{n} D_{n}\right) \Theta_{n}+Q_{n}\right] e^{A_{n}(r-s)} \Gamma_{n} \zeta d s \\
& =\int_{s}^{T} e^{A^{*}(r-s)}\left[P A_{1}+A_{1}^{*} P_{n}+C^{*} P C+\left(P B+C^{\top} P D\right) \Theta+Q\right] e^{A(r-s)} \zeta d r .
\end{aligned}
$$

It follows from (4.27), (4.30), (4.31) and (4.33) that

$$
\begin{aligned}
P(s) \zeta=e^{A^{*}(T-s)} G e^{A(T-s)} \zeta+\int_{s}^{T} e^{A^{*}(r-s)}\left[P A_{1}+A_{1}^{*} P+C^{*} P C\right. \\
\left.+\left(P B+C^{*} P D\right) \Theta+Q\right] e^{A(r-s)} \zeta d r .
\end{aligned}
$$

Step 5. Finally, in this step, we prove that $P(\cdot)$ solves the Riccati equation (2.2). 
From (4.15), we see that

$$
0=B^{*} P+D^{*} P(C+D \Theta)+R \Theta=B^{*} P+D^{*} P C+K \Theta, \quad \text { a.e. }(s, \omega) \in[0, T] \times \Omega .
$$

Consequently,

$$
0=\Theta^{*} B^{*} P+\Theta^{*} D^{*} P C+\Theta^{*} K \Theta, \quad \text { a.e. }(s, \omega) \in[0, T] \times \Omega .
$$

Using (4.36), (4.34) can be written as

$$
\begin{aligned}
P(t) \zeta=e^{(T-s) A} G e^{(T-s) A} \zeta & +\int_{s}^{T} e^{(r-s) A}\left[P A_{1}+A_{1}^{*} P+P B \Theta\right. \\
& \left.+\Theta^{*} B^{*} P+(C+D \Theta)^{*} P(C+D \Theta)+\Theta^{*} R \Theta+Q\right] e^{(r-s) A} \zeta d s .
\end{aligned}
$$

Since $P(T)=G \in \mathbb{S}(H)$, and $Q(\cdot)$ and $R(\cdot)$ are symmetric operator-valued functions, by Proposition 3.1, we have $P(\cdot) \in C_{\mathcal{S}}([0, T] ; \mathbb{S}(H))$. Thus $K(\cdot)$ is a symmetric operator-valued function. Hence, for a.e. $s \in[0, T], K(s)$ admits a generalized pseudo inverse $K(s)^{\dagger}$.

Since $\Lambda_{n}=0$ for all $n \in \mathbb{N}$, we see that

$$
\Pi_{n}-P_{n}\left(C_{n}+D_{n} \Theta_{n}\right)=Z_{n} \widetilde{X}_{n}^{\top}-P_{n}\left(C_{n}+D_{n} \Theta_{n}\right)=0 \quad \text { for every } n \in \mathbb{N} .
$$

Therefore,

$$
\Pi-P(C+D \Theta)=0
$$

This implies that

$$
\left(B^{*} P+D^{*} P C\right)=-K \Theta .
$$

Thus, (2.3) holds and

$$
K^{\dagger}\left(B^{*} P+D^{*} P C\right)=-K^{\dagger} K \Theta .
$$

Noting that $K^{\dagger} K$ is an orthogonal projection, we see that (2.4) holds and

$$
\Theta=-K^{\dagger}\left(B^{*} P+D^{*} P C\right)+\left(I-K^{\dagger} K\right) \theta
$$

for some $\theta(\cdot) \in L^{2}(0, T ; \mathcal{L}(H ; U))$. Consequently,

$$
\left(P B+C^{*} P D\right) \Theta=\Theta^{*} K K^{\dagger}\left(B^{*} P+D^{*} P C\right)=-\left(P B+C^{*} P D\right) K^{\dagger}\left(B^{*} P+D^{*} P C\right) .
$$

From (4.37) and (4.34), we obtain the Riccati equation (2.2). This completes the proof of the "only if" part.

\section{Proof of Theorem 2.2}

In this section, we prove Theorem 2.2. Without loss of generality, we assume that $t=0$. Proof of Theorem 2.2: (i) $\Rightarrow$ (ii). The proof is long. We divide it into three steps.

Step 1. In this step, we introduce a sequence of operator-valued functions $\left\{P_{j}\right\}_{j=1}^{N}$.

Let $P_{0}$ be the solution to

$$
\left\{\begin{array}{l}
\dot{P}_{0}+P_{0}\left(A+A_{1}\right)+\left(A+A_{1}\right)^{*} P_{0}+C^{*} P_{0} C+Q=0 \quad \text { in }[0, T), \\
P_{0}(T)=G
\end{array}\right.
$$


Applying Proposition 3.5 to (5.1) with $\Theta=0$, we obtain that

$$
R(s)+D(s)^{*} P_{0}(s) D(s) \geq \lambda I, \quad P_{0}(s) \geq \alpha I, \quad \text { a.e. } \quad s \in[0, T] .
$$

Inductively, for $j=0,1,2, \cdots$, we set

$$
\begin{aligned}
& K_{j} \triangleq R+D^{*} P_{j} D, \quad L_{j} \triangleq B^{*} P_{j}+D^{*} P_{j} C, \\
& \Theta_{j} \triangleq-K_{j}^{-1} L_{j}, \quad \mathcal{A}_{j} \triangleq A_{1}+B \Theta_{j}, \quad C_{j} \triangleq C+D \Theta_{j},
\end{aligned}
$$

and let $P_{j+1}$ be the solution to

$$
\left\{\begin{array}{l}
\dot{P}_{j+1}+P_{j+1}\left(A+\mathcal{A}_{j}\right)+\left(A+\mathcal{A}_{j}\right)^{*} P_{j+1}+C_{j}^{*} P_{j+1} C_{j}+\Theta_{j}^{*} R \Theta_{j}+Q=0 \quad \text { in }[0, T), \\
P_{j+1}(T)=G .
\end{array}\right.
$$

Step 2. In this step, we show the uniform boundedness of the sequence $\left\{P_{j}\right\}_{j=1}^{\infty}$. From (5.2), we have that

$$
K_{0}(s) \geq \lambda I, \quad P_{0}(s) \geq \alpha I, \quad \text { a.e. } s \in[0, T] .
$$

Then $\Theta_{0}=-K_{0}^{-1} L_{0} \in L^{2}(0, T ; \mathcal{L}(H ; U))$. It follows from Proposition 3.5 (with $P$ and $\Theta$ in (3.17) replaced by $P_{1}$ and $\Theta_{0}$, respectively) that

$$
K_{1}(s) \geq \lambda I, \quad P_{1}(s) \geq \alpha I, \quad \text { a.e. } s \in[0, T] .
$$

Inductively, we have that

$$
K_{j+1}(s) \geq \lambda I, \quad P_{j+1}(s) \geq \alpha I, \quad \text { a.e. } s \in[0, T], \quad j=0,1,2, \cdots .
$$

We now claim that $\left\{P_{j}\right\}_{j=1}^{\infty}$ converges uniformly in $C_{\mathcal{S}}([0, T] ; \mathbb{S}(H))$. To show this, let

$$
\Delta_{j} \triangleq P_{j}-P_{j+1}, \quad \Upsilon_{j} \triangleq \Theta_{j-1}-\Theta_{j}, \quad j \geq 1
$$

Then for $j \geq 1$ and $\zeta \in H$, we have

$$
\begin{aligned}
& -\Delta_{j}(s) \zeta=P_{j+1}(s) \zeta-P_{j}(s) \zeta \\
& =\int_{s}^{T} e^{(r-s) A^{*}}\left[\left(P_{j}(s)-P_{j+1}(s)\right) \mathcal{A}_{j}+\mathcal{A}_{j}^{*}\left(P_{j}(s)-P_{j+1}(s)\right)+C_{j}^{*}\left(P_{j}(s)-P_{j+1}(s)\right) C_{j}\right. \\
& +P_{j}\left(\mathcal{A}_{j-1}-\mathcal{A}_{j}\right)+\left(\mathcal{A}_{j-1}-\mathcal{A}_{j}\right)^{*} P_{j}+C_{j-1}^{*} P_{j} C_{j-1}-C_{j}^{*} P_{j} C_{j}+\Theta_{j-1}^{*} R \Theta_{j-1} \\
& \left.\quad-\Theta_{j}^{*} R \Theta_{j}\right] e^{(r-s) A} \zeta d s \\
& =\int_{s}^{T} e^{(r-s) A^{*}}\left[\Delta_{j}(s) \mathcal{A}_{j}+\mathcal{A}_{j}^{*} \Delta_{j}(s)+C_{j}^{*} \Delta_{j}(s) C_{j}+P_{j}\left(\mathcal{A}_{j-1}-\mathcal{A}_{j}\right)+\left(\mathcal{A}_{j-1}-\mathcal{A}_{j}\right)^{*} P_{j}\right. \\
& + \\
& \left.+C_{j-1}^{*} P_{j} C_{j-1}-C_{j}^{*} P_{j} C_{j}+\Theta_{j-1}^{*} R \Theta_{j-1}-\Theta_{j}^{*} R \Theta_{j}\right] e^{(r-s) A} \zeta d r
\end{aligned}
$$

From (5.3), we have that

$$
\mathcal{A}_{j-1}-\mathcal{A}_{j}=A_{1}+B \Theta_{j-1}-A_{1}-B \Theta_{j}=B\left(\Theta_{j-1}-\Theta_{j}\right)=B \Upsilon_{j},
$$




$$
C_{j-1}-C_{j}=C+D \Theta_{j-1}-C-D \Theta_{j}=D\left(\Theta_{j-1}-\Theta_{j}\right)=D \Upsilon_{j}
$$

and

$$
\begin{aligned}
C_{j-1}^{*} P_{j} C_{j-1}-C_{j}^{*} P_{j} C_{j} \\
=\left(C+D \Theta_{j-1}\right)^{*} P_{j}\left(C+D \Theta_{j-1}\right)-\left(C+D \Theta_{j}\right)^{*} P_{j}\left(C+D \Theta_{j}\right) \\
=\Theta_{j-1}^{*} D^{*} P_{j} D \Theta_{j-1}-\Theta_{j}^{*} D^{*} P_{j} D \Theta_{j}+\Theta_{j-1}^{*} D^{*} P_{j} C+C^{*} P_{j} D \Theta_{j-1}-\Theta_{j}^{*} D^{*} P_{j} C-C^{*} P_{j} D \Theta_{j} \\
=\left(\Theta_{j-1}-\Theta_{j}\right)^{*} D^{*} P_{j} D\left(\Theta_{j-1}-\Theta_{j}\right)+\left(C+D \Theta_{j}\right)^{*} P_{j} D\left(\Theta_{j-1}-\Theta_{j}\right) \\
\quad+\left(\Theta_{j-1}-\Theta_{j}\right)^{*} D^{*} P_{j}\left(C+D \Theta_{j}\right) \\
=\Upsilon_{j}^{*} D^{*} P_{j} D \Upsilon_{j}+C_{j}^{*} P_{j} D \Upsilon_{j}+\Upsilon_{j}^{*} D^{*} P_{j} C_{j} .
\end{aligned}
$$

Similarly, we can obtain that

$$
\left\{\begin{array}{l}
\Theta_{j-1}^{*} R \Theta_{j-1}-\Theta_{j}^{*} R \Theta_{j}=\Upsilon_{j}^{*} R \Upsilon_{j}+\Upsilon_{j}^{*} R \Theta_{j}+\Theta_{j}^{*} R \Upsilon_{j} \\
B^{*} P_{j}+D^{*} P_{j} C_{j}+R \Theta_{j}=B^{*} P_{j}+D^{*} P_{j} C+\left(R+D^{*} P_{j} D\right) \Theta_{j}=0 .
\end{array}\right.
$$

These, together with (5.7), yields that

$$
\begin{aligned}
& \Delta_{j}(s)-\int_{s}^{T} e^{(r-s) A^{*}}\left(\Delta_{j} \mathcal{A}_{j}+\mathcal{A}_{j}^{*} \Delta_{j}+C_{j}^{*} \Delta_{j} C_{j}\right) e^{(r-s) A} d r \\
& =\int_{s}^{T} e^{(r-s) A^{*}}\left(P_{j} B \Upsilon_{j}+\Upsilon_{j}^{*} B^{*} P_{j}+\Upsilon_{j}^{*} D^{*} P_{j} D \Upsilon_{j}+C_{j}^{*} P_{j} D \Upsilon_{j}+\Upsilon_{j}^{*} D^{*} P_{j} C_{j}\right. \\
& \left.\quad+\Upsilon_{j}^{*} R \Upsilon_{j}+\Upsilon_{j}^{*} R \Theta_{j}+\Theta_{j}^{*} R \Upsilon_{j}\right) e^{(r-s) A} d s \\
& =\int_{s}^{T} e^{(r-s) A^{*}}\left[\Upsilon_{j}^{*} K_{j} \Upsilon_{j}+\left(P_{j} B+C_{j}^{*} P_{j} D+\Theta_{j}^{*} R\right) \Upsilon_{j}+\Upsilon_{j}^{*}\left(B^{*} P_{j}+D^{*} P_{j} C_{j}+R \Theta_{j}\right)\right] e^{(r-s) A} d r \\
& =\int_{s}^{T} e^{A^{*}(r-s)} \Upsilon_{j}^{*} K_{j} \Upsilon_{j} e^{A(r-s)} d r .
\end{aligned}
$$

From (5.8), we know that $\Delta_{j}(\cdot)$ is a solution to $(\underline{3.28})$ with $\widetilde{G}=0, \widetilde{A}=\mathcal{A}_{j}, \widetilde{C}=\mathcal{C}_{j}$ and $\widetilde{Q}=\Upsilon_{j}^{*} K_{j} \Upsilon_{j} \geq 0$. Using Lemma 3.6, we have that $\Delta_{j}(\cdot) \geq 0$, namely, $P_{j-1}(\cdot)-P_{j}(\cdot) \geq 0$ for $j \geq 1$. Noting (5.6), we obtain

$$
P_{1}(s) \geq P_{j}(s) \geq P_{j+1}(s) \geq \alpha I, \quad \forall s \in[0, T], \quad \forall j \geq 1 .
$$

Therefore, the sequence $\left\{P_{j}\right\}_{j=1}^{\infty}$ is uniformly bounded. Consequently, there exists a constant $\mathcal{C}>0$ such that (noting (5.6) ) for all $j \geq 0$ and a.e. $s \in[0, T]$,

$$
\left\{\begin{array}{l}
\left|P_{j}(s)\right|_{\mathcal{L}(H)} \leq \mathcal{C}, \quad\left|K_{j}(s)\right|_{\mathcal{L}(U)} \leq \mathcal{C} \\
\left|\Theta_{j}(s)\right|_{\mathcal{L}(H ; U)} \leq \mathcal{C}\left(|B(s)|_{\mathcal{L}(U ; H)}+|C(s)|_{\mathcal{L}(H)}\right) \\
\left|\mathcal{A}_{j}(s)\right|_{\mathcal{L}(H)} \leq\left|A_{1}(s)\right|_{\mathcal{L}(H)}+\mathcal{C}|B(s)|_{\mathcal{L}(U ; H)}\left(|B(s)|_{\mathcal{L}(U ; H)}+|C(s)|_{\mathcal{L}(H)}\right) \\
\left|C_{j}(s)\right|_{\mathcal{L}(H)} \leq \mathcal{C}\left(|B(s)|_{\mathcal{L}(U ; H)}+|C(s)|_{\mathcal{L}(H)}\right)
\end{array}\right.
$$

Step 3. In this step, we prove the convergence of the sequence $\left\{P_{j}\right\}_{j=1}^{\infty}$. 
Noting

$$
\Lambda_{j}=\Theta_{j-1}-\Theta_{j}=K_{j}^{-1} D^{*} \Delta_{j-1} D K_{j-1}^{-1} L_{j}-K_{j-1}^{-1}\left(B^{*} \Delta_{j-1}+D^{*} \Delta_{j-1} C\right)
$$

and (5.9), one has

$$
\begin{aligned}
& \left|\Upsilon_{j}(s)^{*} K_{j}(s) \Upsilon_{j}(s)\right|_{\mathcal{L}(H)} \\
& \leq\left(\left|\Theta_{j}(s)\right|_{\mathcal{L}(U)}+\left|\Theta_{j-1}(s)\right|_{\mathcal{L}(U)}\right)\left|K_{j}(s)\right|_{\mathcal{L}(U)}\left|\Theta_{j-1}(s)-\Theta_{j}(s)\right|_{\mathcal{L}(U)} \\
& \leq \mathcal{C}\left(|B(s)|_{\mathcal{L}(U ; H)}+|C(s)|_{\mathcal{L}(H)}\right)^{2}\left|\Delta_{j-1}(s)\right|_{\mathcal{L}(H)} .
\end{aligned}
$$

Equation (5.8) implies that

$$
\Delta_{j}(s)=-\int_{s}^{T} e^{(r-s) A^{*}}\left(\Delta_{j} \mathcal{A}_{j}+\mathcal{A}_{j}^{*} \Delta_{j}+C_{j}^{*} \Delta_{i} C_{j}+\Upsilon_{j}^{*} K_{j} \Upsilon_{j}\right) e^{(r-s) A} d r
$$

Making use of (5.10) and noting (5.9), we get

$$
\left|\Delta_{j}(s)\right|_{\mathcal{L}(H)} \leq \int_{s}^{T} \varphi(r)\left(\left|\Delta_{j}(r)\right|_{\mathcal{L}(H)}+\left|\Delta_{j-1}(r)\right|_{\mathcal{L}(H)}\right) d r, \quad \forall s \in[0, T], \quad \forall j \geq 1,
$$

where $\varphi(\cdot)$ is a nonnegative integrable function independent of $\Delta_{j}(\cdot)$. By Gronwall's inequality,

$$
\left|\Delta_{j}(s)\right|_{\mathcal{L}(H)} \leq e^{\int_{0}^{T} \varphi(r) d r} \int_{s}^{T} \varphi(r)\left|\Delta_{j-1}(r)\right|_{\mathcal{L}(H)} d r \equiv b \int_{s}^{T} \varphi(r)\left|\Delta_{j-1}(r)\right|_{\mathcal{L}(H)} d r,
$$

where $b=e^{\int_{0}^{T} \varphi(r) d r}$. Set $a \triangleq \max _{0 \leq r \leq T}\left|\Delta_{0}(r)\right|_{\mathcal{L}(H)}$. By induction, we deduce that

$$
\left|\Delta_{j}(s)\right|_{\mathcal{L}(H)} \leq a \frac{b^{j}}{j !}\left(\int_{s}^{T} \varphi(r) d r\right)^{j}, \quad \forall s \in[0, T],
$$

which implies the uniform convergence of $\left\{P_{j}\right\}_{j=1}^{\infty}$. Denote by $P$ the limit of $\left\{P_{j}\right\}_{j=1}^{\infty}$, then (noting (5.6) $)$

$$
K(s)=\lim _{j \rightarrow \infty} K_{j}(s) \geq \lambda I, \quad \text { a.e. } \quad s \in[0, T]
$$

and as $j \rightarrow \infty$,

$$
\left\{\begin{array}{l}
\Theta_{j} \rightarrow-K^{-1} L \equiv \Theta \quad \text { in } L^{2}(0, T ; \mathcal{L}(H ; U)), \\
\mathcal{A}_{j} \rightarrow A_{1}+B \Theta \quad \text { in } L^{1}(0, T ; \mathcal{L}(H)), \quad C_{j} \rightarrow C+D \Theta \quad \text { in } L^{2}(0, T ; \mathcal{L}(H)) .
\end{array}\right.
$$

Therefore, $P(\cdot)$ solves the following equation (in the sense of mild solution):

$$
\left\{\begin{array}{l}
\dot{P}+P(A+B \Theta)+(A+B \Theta)^{*} P+(C+D \Theta)^{*} P(C+D \Theta)+\Theta^{*} R \Theta+Q=0 \quad \text { in }[0, T), \\
P(T)=G,
\end{array}\right.
$$

which is equivalent to (2.2).

(ii) $\Rightarrow$ (i). Let $P(\cdot)$ be the strongly regular solution to (2.2). Then there exists a $\lambda>0$ such that

$$
K(s) \geq \lambda I, \quad \text { a.e. } s \in[0, T] .
$$


Set $\Theta \triangleq-K^{-1} L \in L^{2}(0, T ; \mathcal{L}(H ; U))$. For any $u(\cdot) \in \mathcal{U}[0, T]$, let $x(\cdot)=x(\cdot ; 0,0, u)$ be the solution to (1.1) with $t=0$ and $\eta=0$. Applying Itô's formula to $s \mapsto\langle P(s) x(s), x(s)\rangle$, we have

$$
\begin{aligned}
& \mathcal{J}(0,0 ; u(\cdot))=\mathbb{E}\left[\langle G x(T), x(T)\rangle+\int_{0}^{T}(\langle Q x, x\rangle+\langle R u, u\rangle) d s\right] \\
& =\mathbb{E} \int_{0}^{T}\left\{\left\langle-\left[P\left(A+A_{1}\right)+\left(A+A_{1}\right)^{*} P+C^{*} P C+Q-L^{*} K^{\dagger} L\right] x, x\right\rangle\right. \\
& \quad+\left\langle P\left[\left(A+A_{1}\right) x+B u\right], x\right\rangle+\left\langle P x,\left(A+A_{1}\right) x+B u\right\rangle \\
& \quad+\langle P(C x+D u), C x+D u\rangle+\langle Q x, x\rangle+\langle R u, u\rangle\} d s \\
& =\mathbb{E} \int_{0}^{T}\left(\left\langle\Theta^{*} K \Theta x, x\right\rangle-2\langle K \Theta x, u\rangle+\langle K u, u\rangle\right) d s \\
& =\mathbb{E} \int_{0}^{T}\langle K(u-\Theta x), u-\Theta x\rangle d s .
\end{aligned}
$$

Noting (5.11) and making use of Lemma 3.7, we obtain that for some $c_{0}>0$ and all $u(\cdot) \in$ $\mathcal{U}[0, T]$,

$$
\mathcal{J}(0,0 ; u(\cdot))=\mathbb{E} \int_{0}^{T}\langle K(u-\Theta x), u-\Theta x\rangle d s \geq c_{0} \lambda \mathbb{E} \int_{0}^{T}|u(s)|^{2} d s .
$$

Then (i) holds.

Remark 5.1 From the first part of the proof of Theorem 2.2, we see that if (2.9) holds, then the strongly regular solution to (2.2) satisfies (2.6) with the same constant $\lambda>0$.

\section{The uniform convexity of the cost functional}

In this section, we study the uniform convexity of the cost functional.

Define four operators as follows:

$$
\Gamma_{t}: H \rightarrow \mathcal{X}[t, T] \triangleq L_{\mathbb{F}}^{2}(t, T ; H), \quad \Gamma_{t} \eta=x(\cdot ; t, \eta, 0), \quad \forall \eta \in H,
$$

where $x(\cdot ; t, \eta, 0)$ is the solution to (1.1) with $u \equiv 0$;

$$
\Xi_{t}: \mathcal{U}[t, T] \rightarrow \mathcal{X}[t, T], \quad \Xi_{t} u=x(\cdot ; t, 0, u), \quad \forall u \in \mathcal{U}[t, T],
$$

where $x(\cdot ; t, 0, u)$ is the solution to (1.1) with $\eta=0$;

$$
\widehat{\Gamma}_{t}: H \rightarrow L_{\mathcal{F}_{T}}^{2}(\Omega ; H), \quad \widehat{\Gamma}_{t} \eta=x(T ; t, \eta, 0), \quad \forall \eta \in H ;
$$

and

$$
\widehat{\Xi}_{t}: \mathcal{U}[t, T] \rightarrow L_{\mathcal{F}_{T}}^{2}(\Omega ; H), \quad \widehat{\Xi}_{t} u=x(T ; t, 0, u), \quad \forall \eta \in H .
$$

From the well-posedness of (1.1), we find that all these operators are bounded linear operators. Then, for any $t \in[0, T)$ and $(\eta, u(\cdot)) \in H \times \mathcal{U}[t, T]$, the corresponding state process $x(\cdot)$ and its terminal value $x(T)$ are given by

$$
x(\cdot)=\left(\Gamma_{t} \eta\right)(\cdot)+\left(\Xi_{t} u\right)(\cdot), \quad x(T)=\widehat{\Gamma}_{t} \eta+\widehat{\Xi}_{t} u .
$$


Hence, the cost functional can be written as

$$
\mathcal{J}(t, \eta ; u(\cdot))=\left\langle G\left(\widehat{\Gamma}_{t} \eta+\widehat{\Xi}_{t} u\right), \widehat{\Gamma}_{t} \eta+\widehat{\Xi}_{t} u\right\rangle+\left\langle Q\left(\Gamma_{t} \eta+\Xi_{t} u\right), \Gamma_{t} \eta+\Xi_{t} u\right\rangle+\langle R u, u\rangle .
$$

Recall that $u(\cdot) \mapsto \mathcal{J}(t, \eta ; u(\cdot))$ is uniformly convex if and only if for some $\lambda>0$,

$$
\mathcal{J}(t, 0 ; u(\cdot)) \geq \lambda \mathbb{E} \int_{t}^{T}|u(s)|^{2} d s, \quad \forall u(\cdot) \in \mathcal{U}[t, T] .
$$

From (6.1), (6.2) is equivalent to the following:

$$
\widehat{\Xi}_{t}^{*} G \widehat{\Xi}_{t}+\Xi_{t}^{*} Q \Xi_{t}+R \geq \lambda I, \quad \text { for some } \lambda>0 \text {. }
$$

According to the above argument, we get the following result:

Proposition 6.1 The map $u(\cdot) \mapsto \mathcal{J}(t, \eta ; u(\cdot))$ is uniformly convex if and only if (6.3) holds.

It is obvious that if the condition (1.4) hold, then (6.3) holds for $\lambda=\delta$. On the other hand, if $R \geq \delta I$ does not hold, (6.3) still may be true if $G$ is large enough. An example is given below:

We first introduce the following assumption.

(AS3) Assume that $D$ is invertible for a.e. $t \in[0, T]$ and $D(\cdot)^{-1} \in L^{\infty}(0, T ; \mathcal{L}(H ; U)$ ).

Without loss of generality, we set $t=0$ in (1.1). Under (AS3), the control system (1.1) can be written as a BSEE

$$
\left\{\begin{array}{l}
d x=\left[\left(A+A_{1}\right) x+B D^{-1} \hat{x}\right] d s+(C x+\hat{x}) d W(s) \quad \text { in }[0, T), \\
x(T) \in L_{\mathcal{F}_{T}}^{2}(\Omega ; H),
\end{array}\right.
$$

where $\hat{x}(\cdot)=D(\cdot) u(\cdot)$ and $x(T)$ is the value of the solution to (1.1) at time $T$. If (AS1) and (AS3) hold, then (6.4) is well-posed and

$$
|(x(\cdot), \hat{x}(\cdot))|_{C_{\mathbb{F}}\left([0, T] ; L^{2}(\Omega ; H)\right) \times L_{\mathbb{F}}^{2}(0, T ; H)} \leq \mathcal{C}|x(T)|_{L_{\mathcal{F}_{T}}^{2}(\Omega ; H)} .
$$

This implies that there is a $\mathcal{C}_{0}>0$, depending only on $A, A_{1}, B, C$ and $D$ such that

$$
|u(\cdot)|_{L_{\mathbb{F}}^{2}(0, T ; H)}^{2} \leq \mathcal{C}_{0}|x(T)|_{L_{\mathcal{F}_{T}}^{2}(\Omega ; H)}^{2} \cdot
$$

Let us make the following further assumption.

(AS4) There is a $\mu_{0}>\mathcal{C}_{0}\left(|R|_{L^{\infty}(0, T ; \mathcal{L}(U))}+\varepsilon_{0}\right)$ with $\varepsilon_{0}>0$ such that for any $\zeta \in H$, $\langle G \zeta, \zeta\rangle \geq \mu_{0}|\zeta|^{2}$.

If (AS4) holds, it follows from (6.5) that

$$
\left\langle\widehat{\Xi}_{t}^{*} G \widehat{\Xi}_{t} u, u\right\rangle=\left\langle G \widehat{\Xi}_{t} u, \hat{\Xi}_{t} u\right\rangle \geq \mu_{0}|u|_{L_{\mathbb{F}}^{2}(0, T ; H)}^{2} \geq\left(|R|_{L^{\infty}(0, T ; \mathcal{L}(U))}+\varepsilon_{0}\right)|u|_{L_{\mathbb{F}}^{2}(0, T ; H)}^{2} .
$$

This deduces that (6.3) holds for $\lambda=\varepsilon_{0}$.

According to the above argument, we have the following result.

Theorem 6.1 Let (AS1), (AS3) and (AS4) hold. Then the map $u(\cdot) \mapsto \mathcal{J}(t, \eta ; u(\cdot))$ is uniformly convex. 


\section{Appendix: Proofs for some preliminary results}

\subsection{Proof of Lemma 3.1}

Proof of Lemma 3.1: Without loss of generality, we assume that $t=0$. Write

$$
N=\left\lceil\frac{1}{\varepsilon}\left(|\mathcal{A}|_{L^{1}(0, T ; \mathcal{L}(H))}^{2}+|\mathcal{B}|_{L^{2}(0, T ; \mathcal{L}(H))}^{2}\right)\right\rceil+1
$$

where $\varepsilon>0$ is a constant to be determined later. Define a sequence of $\left\{\tau_{j, \varepsilon}\right\}_{j=1}^{N}$ as follows:

$$
\left\{\begin{aligned}
\tau_{1, \varepsilon}=\left\{\left.r \in[0, T]\left|\left(\int_{0}^{r}|\mathcal{A}(s)|_{\mathcal{L}(H)} d s\right)^{2}+\int_{0}^{r}\right| \mathcal{B}(s)\right|_{\mathcal{L}(H)} ^{2} d s=\varepsilon\right\} \\
\tau_{k, \varepsilon}=\left\{\left.r \in[0, T]\left|\left(\int_{\tau_{k-1, \varepsilon}}^{r}|\mathcal{A}(s)|_{\mathcal{L}(H)} d s\right)^{2}+\int_{\tau_{k-1, \varepsilon}}^{r}\right| \mathcal{B}(s)\right|_{\mathcal{L}(H)} ^{2} d s=\varepsilon\right\}, \\
k=2, \cdots, N .
\end{aligned}\right.
$$

Consider the following SEE:

$$
\left\{\begin{array}{l}
d x=(A x+\tilde{f}) d s+\tilde{g} d W(s) \quad \text { in }(0, T] \\
x(0)=\eta
\end{array}\right.
$$

Here $\tilde{f} \in L_{\mathbb{F}}^{2}\left(\Omega ; L^{1}(0, T ; H)\right)$ and $\tilde{g} \in L_{\mathbb{F}}^{2}(0, T ; H)$. Clearly, (7.1) admits a unique mild solution $x \in C_{\mathbb{F}}\left([0, T] ; L^{2}(\Omega ; H)\right)$ (e.g. [12, Chapter 6$\left.]\right)$. Define a map $\mathcal{J}: C_{\mathbb{F}}\left(\left[0, \tau_{1, \varepsilon}\right] ; L^{2}(\Omega ; H)\right) \rightarrow$ $C_{\mathbb{F}}\left(\left[0, \tau_{1, \varepsilon}\right] ; L^{2}(\Omega ; H)\right)$ as follows:

$$
C_{\mathbb{F}}\left(\left[0, \tau_{1, \varepsilon}\right] ; L^{2}(\Omega ; H)\right) \ni \tilde{x} \mapsto x=\mathcal{J}(\tilde{x}),
$$

where $x$ is the solution to (7.1) with $\tilde{f}$ and $\tilde{g}$ replaced by $\mathcal{A} \tilde{x}+f$ and $\mathcal{B} \tilde{x}+g$, respectively. We claim that $\mathcal{J}$ is contractive. Indeed, for any $\tilde{x}_{1}, \tilde{x}_{2} \in C_{\mathbb{F}}\left(\left[0, \tau_{1, \varepsilon}\right] ; L^{2}(\Omega ; H)\right)$,

$$
\begin{aligned}
& \left|\mathcal{J}\left(\tilde{x}_{1}\right)-\mathcal{J}\left(\tilde{x}_{1}\right)\right|_{C_{\mathbb{F}}\left(\left[0, \tau_{1, \varepsilon}\right] ; L^{2}(\Omega ; H)\right)}^{2} \\
\leq & 2 \sup _{s \in\left[0, \tau_{1, \varepsilon}\right]} \mathbb{E}\left(\left|\int_{0}^{s} e^{(s-r) A} \mathcal{A}\left(\tilde{x}_{1}-\tilde{x}_{2}\right) d r\right|_{H}\right)^{2}+\left.\left.2 \mathbb{E}\left|\int_{0}^{\tau_{1, \varepsilon}}\right| e^{(s-r) A} \mathcal{B}\left(\tilde{x}_{1}-\tilde{x}_{2}\right)\right|_{H} ^{2} d W(r)\right|_{H} \\
\leq & \left.\left.2\left(\sup _{s \in[0, T]}\left|e^{A s}\right|_{\mathcal{L}(H)}^{2}\right)|| \mathcal{A}\right|_{\mathcal{L}(H)}\right|_{L^{1}\left(0, \tau_{1, \varepsilon}\right)} ^{2}\left|\tilde{x}_{1}-\tilde{x}_{2}\right|_{C_{\mathbb{F}}\left(\left[0, \tau_{1, \varepsilon}\right] ; L^{2}(\Omega ; H)\right)}^{2} \\
& +\left.\left.2\left(\sup _{s \in[0, T]}\left|e^{A s}\right|_{\mathcal{L}(H)}^{2}\right)|| \mathcal{B}\right|_{\mathcal{L}(H)}\right|_{L^{2}\left(0, \tau_{1, \varepsilon}\right)} ^{2}\left|\tilde{x}_{1}-\tilde{x}_{2}\right|_{C_{\mathbb{F}}\left(\left[0, \tau_{1, \varepsilon}\right] ; L^{2}(\Omega ; H)\right)}^{2} .
\end{aligned}
$$

Let

$$
M_{T}=\sup _{s \in[0, T]}\left|e^{A s}\right|_{\mathcal{L}(H)}, \quad \varepsilon=\frac{1}{16 M_{T}^{2}}
$$

It follows from (7.2) that

$$
\left|\mathcal{J}\left(\tilde{x}_{1}\right)-\mathcal{J}\left(\tilde{x}_{1}\right)\right|_{C_{\mathbb{F}}\left(\left[0, \tau_{1, \varepsilon}\right] ; L^{2}(\Omega ; H)\right)}^{2} \leq \frac{1}{4}\left|\tilde{x}_{1}-\tilde{x}_{2}\right|_{C_{\mathbb{F}}\left(\left[0, \tau_{1, \varepsilon}\right] ; L^{2}(\Omega ; H)\right)}^{2} .
$$


This shows that $\mathcal{J}$ is contractive. Hence, it has a unique fixed point $x \in C_{\mathbb{F}}\left(\left[0, \tau_{1, \varepsilon}\right] ; L^{2}(\Omega ; H)\right)$, which solves (3.1) in $\left[0, \tau_{1, \varepsilon}\right]$ (in the sense of mild solution). Inductively, we conclude that (3.1) admits a mild solution $x$ in $\left[\tau_{k-1, \varepsilon}, \tau_{k, \varepsilon}\right]$ for $k=2, \cdots, N$. Furthermore,

$$
\begin{aligned}
& |x|_{C_{\mathbb{F}}\left(\left[0, \tau_{1, \varepsilon}\right] ; L^{2}(\Omega ; H)\right)}^{2} \\
\leq & 4 \sup _{s \in\left[0, \tau_{1, \varepsilon}\right]} \mathbb{E}\left(\left|\int_{0}^{s} e^{A(t-r)} \mathcal{A} x d r\right|_{H}\right)^{2}+4 \sup _{t \in\left[0, \tau_{1, \varepsilon}\right]} \mathbb{E}\left(\left|\int_{0}^{s} e^{A(s-r)} \mathcal{B} x d W(r)\right|_{H}\right)^{2} \\
& +4 \sup _{s \in[0, T]} \mathbb{E}\left(\left|e^{A s} \eta+\int_{0}^{s} e^{A(s-r)} f d r+\int_{0}^{t} e^{A(s-r)} g d W(r)\right|_{H}\right)^{2} \\
\leq & \left.4 M_{T}^{2}|| \mathcal{A}||_{\mathcal{L}(H)}\right|_{L^{1}\left(0, \tau_{1, \varepsilon}\right)} ^{2}|x|_{C_{\mathbb{F}}\left(\left[0, \tau_{1, \varepsilon}\right] ; L^{2}(\Omega ; H)\right)}^{2}+\left.\left.4 M_{T}^{2}|| \mathcal{B}\right|_{\mathcal{L}(H)}\right|_{L^{2}\left(0, \tau_{1, \varepsilon}\right)} ^{2}|x|_{C_{\mathbb{F}}\left(\left[0, \tau_{1, \varepsilon}\right] ; L^{2}(\Omega ; H)\right)}^{2} \\
& +\mathcal{C}\left(|\eta|_{H}^{2}+|f|_{L_{\mathbb{F}}^{2}\left(\Omega ; L^{1}(0, T ; H)\right)}^{2}+|g|_{L_{\mathbb{F}}^{2}(0, T ; H)}^{2}\right) .
\end{aligned}
$$

This, together with the choice of $\tau_{1, \varepsilon}$, implies that

$$
|x|_{C_{\mathbb{F}}\left(\left[0, \tau_{1, \varepsilon}\right] ; L^{2}(\Omega ; H)\right)}^{2} \leq \mathcal{C}\left(|\eta|_{H}^{2}+|f|_{L_{\mathbb{F}}^{2}\left(\Omega ; L^{1}(0, T ; H)\right)}^{2}+|g|_{L_{\mathbb{F}}^{2}(0, T ; H)}^{2}\right) .
$$

Repeating the above argument, we obtain (3.2). The uniqueness of the solution is obvious.

\subsection{Proof of Lemma 3.2}

Proof of Lemma 3.2: Without loss of generality, we assume that $t=0$.

Write

$$
N=\left\lceil\frac{1}{\varepsilon}\left(\left|A_{1}^{*}\right|_{L^{1}(t, T ; \mathcal{L}(H))}^{2}+\left|C^{*}\right|_{L^{2}(t, T ; \mathcal{L}(H))}^{2}\right)\right\rceil+1
$$

where $\varepsilon>0$ is a constant to be determined later. Define a sequence of $\left\{\tau_{k, \varepsilon}\right\}_{k=1}^{N}$ as follows:

$$
\left\{\begin{array}{l}
\tau_{1, \varepsilon}=\left\{\left.r \in[0, T]\left|\left(\int_{r}^{T}\left|A_{1}(s)^{*}\right|_{\mathcal{L}(H)} d s\right)^{2}+\int_{r}^{T}\right| C(s)^{*}\right|_{\mathcal{L}(H)} ^{2} d s=\varepsilon\right\}, \\
\tau_{k, \varepsilon}=\left\{\left.r \in[0, T]\left|\left(\int_{r}^{\tau_{k-1, \varepsilon}}\left|A_{1}(s)^{*}\right|_{\mathcal{L}(H)} d s\right)^{2}+\int_{r}^{\tau_{k-1, \varepsilon}}\right| C(s)^{*}\right|_{\mathcal{L}(H)} ^{2} d s=\varepsilon\right\},
\end{array} k=2, \cdots, N .\right.
$$

Consider the following BSEE:

$$
\left\{\begin{array}{l}
d y=-\left(A^{*} y+\tilde{h}\right) d s+z d W(s) \quad \text { in }[0, T) \\
y(T)=\xi
\end{array}\right.
$$

where $\tilde{h} \in L_{\mathbb{F}}^{2}\left(\Omega ; L^{1}(0, T ; H)\right)$. Clearly, (17.7) admits a unique mild solution $(y(\cdot), z(\cdot)) \in$ $C_{\mathbb{F}}\left([0, T] ; L^{2}(\Omega ; H)\right) \times L_{\mathbb{F}}^{2}(0, T ; H)($ e.g. [25]) such that

$$
y(s)=e^{A^{*}(T-s)} \xi+\int_{s}^{T} e^{A^{*}(r-s)} \tilde{h}(r) d r-\int_{s}^{T} e^{A^{*}(r-s)} z(r) d W(r),
$$

and

$$
z(s)=e^{A^{*}(T-s)} l(s)+\int_{s}^{T} e^{A^{*}(\eta-s)} \kappa(\eta, s) d \eta,
$$


where $l(\cdot) \in L_{\mathbb{F}}^{2}(0, T ; H)$ such that

$$
\mathbb{E}\left(\xi \mid \mathcal{F}_{s}\right)=\mathbb{E} \xi+\int_{0}^{s} l(\sigma) d W(\sigma)
$$

and $\kappa(\cdot, \cdot) \in L^{1}\left(0, T ; L_{\mathbb{F}}^{2}(0, T ; H)\right)$ such that for a.e. $s \in[0, T]$,

$$
\tilde{h}(s)=\mathbb{E} \tilde{h}(s)+\int_{0}^{s} \kappa(s, \sigma) d W(\sigma)
$$

and

$$
|\kappa(\cdot, \cdot)|_{L^{1}\left(r, T ; L_{\mathbb{F}}^{2}(r, T ; H)\right)} \leq|\tilde{h}(\cdot)|_{L_{\mathbb{F}}^{1}\left(r, T ; L^{2}(\Omega ; H)\right)} .
$$

Define a map $\mathcal{J}: \quad L_{\mathbb{F}}^{2}\left(\Omega ; C\left(\left[\tau_{1, \varepsilon}, T\right] ; H\right)\right) \times L_{\mathbb{F}}^{2}\left(\tau_{1, \varepsilon}, T ; H\right) \rightarrow L_{\mathbb{F}}^{2}\left(\Omega ; C\left(\left[\tau_{1, \varepsilon}, T\right] ; H\right)\right) \times$ $L_{\mathbb{F}}^{2}\left(\tau_{1, \varepsilon}, T ; H\right)$ as follows:

$$
L_{\mathbb{F}}^{2}\left(\Omega ; C\left(\left[\tau_{1, \varepsilon}, T\right] ; H\right)\right) \times L_{\mathbb{F}}^{2}\left(\tau_{1, \varepsilon}, T ; H\right) \ni(\tilde{y}, \tilde{z}) \mapsto(y, z)=\mathcal{J}(\tilde{y}, \tilde{z}),
$$

where $(y, z)$ is the solution to (17.7) with $\tilde{h}$ replaced by $A_{1}^{*} \tilde{y}+C^{*} \tilde{z}+h$. We claim that $\mathcal{J}$ is contractive. Indeed, for $j=1,2$ and $\left(\tilde{y}_{j}, \tilde{z}_{j}\right) \in L_{\mathbb{F}}^{2}\left(\Omega ; C\left(\left[\tau_{1, \varepsilon}, T\right] ; H\right)\right) \times L_{\mathbb{F}}^{2}\left(\tau_{1, \varepsilon}, T ; H\right)$, denote by $\tilde{h}_{j}=A_{1}^{*} \tilde{y}_{j}+C^{*} \tilde{z}_{j}+h$ and $\kappa_{j}(\cdot, \cdot) \in L^{1}\left(0, T ; L_{\mathbb{F}}^{2}(0, T ; H)\right)$ such that for a.e. $s \in[0, T]$,

$$
\tilde{h}_{j}(s)=\mathbb{E} \tilde{h}_{j}(s)+\int_{0}^{s} \kappa_{j}(s, \sigma) d W(\sigma)
$$

and

$$
\left|\kappa_{j}(\cdot, \cdot)\right|_{L^{1}\left(r, T ; L_{\mathbb{F}}^{2}(r, T ; H)\right)} \leq\left|\tilde{h}_{j}(\cdot)\right|_{L_{\mathbb{F}}^{1}\left(r, T ; L^{2}(\Omega ; H)\right)} \cdot
$$

Let $\widetilde{M}_{T}=\sup _{s \in[0, T]}\left|e^{A^{*} s}\right|_{\mathcal{L}(H)}$. Then

$$
\begin{aligned}
& \left|\tilde{z}_{1}-\tilde{z}_{2}\right|_{L_{\mathbb{F}}^{2}\left(\tau_{1, \varepsilon}, T ; H\right)}^{2} \\
= & \mathbb{E} \int_{\tau_{1, \varepsilon}}^{T}\left|\int_{s}^{T} e^{A^{*}(r-s)}\left(\kappa_{1}(r, s)-\kappa_{2}(r, s)\right) d r\right|_{H}^{2} d s \\
\leq & \widetilde{M}_{T}^{2}\left[\left(\int_{\tau_{1, \varepsilon}}^{T}\left|A_{1}(s)^{*}\right|_{\mathcal{L}(H)} d s\right)^{2}+\int_{\tau_{1, \varepsilon}}^{T}\left|C(s)^{*}\right|_{\mathcal{L}(H)}^{2} d s\right] \\
& \times\left(\left|\tilde{y}_{1}-\tilde{y}_{2}\right|_{L_{\mathbb{F}}^{2}\left(\Omega ; C\left(\left[\tau_{1, \varepsilon}, T\right] ; H\right)\right)}^{2}+\left|\tilde{z}_{1}-\tilde{z}_{2}\right|_{L_{\mathbb{F}}^{2}\left(\tau_{1, \varepsilon}, T ; H\right)}^{2}\right) \\
\leq & \widetilde{M}_{T}^{2} \varepsilon\left(\left|\tilde{y}_{1}-\tilde{y}_{2}\right|_{L_{\mathbb{P}}^{2}\left(\Omega ; C\left(\left[\tau_{1, \varepsilon}, T\right] ; H\right)\right)}^{2}+\left|\tilde{z}_{1}-\tilde{z}_{2}\right|_{L_{\mathbb{F}}^{2}\left(\tau_{1, \varepsilon}, T ; H\right)}^{2}\right)
\end{aligned}
$$

and

$$
\begin{aligned}
& \left|\tilde{y}_{1}-\tilde{y}_{2}\right|_{L_{\mathbb{F}}^{2}\left(\Omega ; C\left(\left[\tau_{1, \varepsilon}, T\right] ; H\right)\right)}^{2} \\
& \leq\left.\left. 2 \mathbb{E}\left|\int_{\tau_{1, \varepsilon}}^{T}\right| e^{A^{*}\left(s-\tau_{1, \varepsilon}\right)}\left(\tilde{h}_{1}(s)-\tilde{h}_{2}(s)\right)\right|_{H} d s\right|^{2}+2 \mathbb{E} \int_{\tau_{1, \varepsilon}}^{T}\left|e^{A^{*}\left(s-\tau_{1, \varepsilon}\right)}\left(z_{1}(s)-z_{2}(s)\right)\right|_{H}^{2}(\mathbb{A} s \\
& \leq 2 \widetilde{M}_{T}^{2}\left|\tilde{h}_{1}(\cdot)-\tilde{h}_{2}(\cdot)\right|_{L_{\mathbb{F}}^{1}\left(\tau_{1, \varepsilon}, T ; L^{2}(\Omega ; H)\right)}^{2}+2 \widetilde{M}_{T}^{2}\left|\tilde{z}_{1}-\tilde{z}_{2}\right|_{L_{\mathbb{F}}^{2}\left(\tau_{1, \varepsilon}, T ; H\right)}^{2} \\
& \leq 2\left(\widetilde{M}_{T}^{2}+\widetilde{M}_{T}^{4}\right) \varepsilon\left(\left|\tilde{y}_{1}-\tilde{y}_{2}\right|_{L_{\mathbb{F}}^{2}\left(\Omega ; C\left(\left[\tau_{1, \varepsilon}, T\right] ; H\right)\right)}^{2}+\left|\tilde{z}_{1}-\tilde{z}_{2}\right|_{L_{\mathbb{F}}^{2}\left(\tau_{1, \varepsilon}, T ; H\right)}^{2}\right) \\
& 28
\end{aligned}
$$


Thus,

$$
\begin{aligned}
& \left|\mathcal{J}\left(\tilde{y}_{1}, \tilde{z}_{1}\right)-\mathcal{J}\left(\tilde{y}_{2}, \tilde{z}_{2}\right)\right|_{L_{\mathbb{F}}^{2}\left(\Omega ; C\left(\left[\tau_{1, \varepsilon}, T\right] ; H\right)\right) \times L_{\mathbb{F}}^{2}\left(\tau_{1, \varepsilon}, T ; H\right)}^{2} \\
\leq & \left(3 \widetilde{M}_{T}^{2}+2 \widetilde{M}_{T}^{4}\right) \varepsilon\left(\left|\tilde{y}_{1}-\tilde{y}_{2}\right|_{L_{\mathbb{P}}^{2}\left(\Omega ; C\left(\left[\tau_{1, \varepsilon}, T\right] ; H\right)\right)}^{2}+\left|\tilde{z}_{1}-\tilde{z}_{2}\right|_{L_{\mathbb{F}}^{2}\left(\tau_{1, \varepsilon}, T ; H\right)}^{2}\right) .
\end{aligned}
$$

Let $\varepsilon=\frac{1}{4\left(3 \widetilde{M}_{T}^{2}+2 \widetilde{M}_{T}^{4}\right)}$. It follows from $(7.20)$ that

$$
\begin{aligned}
& \left|\mathcal{J}\left(\tilde{y}_{1}, \tilde{z}_{1}\right)-\mathcal{J}\left(\tilde{y}_{2}, \tilde{z}_{2}\right)\right|_{L_{\mathbb{F}}^{2}\left(\Omega ; C\left(\left[\tau_{1, \varepsilon}, T\right] ; H\right)\right) \times L_{\mathbb{F}}^{2}\left(\tau_{1, \varepsilon}, T ; H\right)}^{2} \\
& \leq \frac{1}{4}\left|\left(\tilde{y}_{1}, \tilde{z}_{1}\right)-\left(\tilde{y}_{2}, \tilde{z}_{2}\right)\right|_{L_{\mathbb{F}}^{2}\left(\Omega ; C\left(\left[\tau_{1, \varepsilon}, T\right] ; H\right)\right) \times L_{\mathbb{F}}^{2}\left(\tau_{1, \varepsilon}, T ; H\right)}^{2}
\end{aligned}
$$

Consequently, $\mathcal{J}$ is contractive. Hence, it has a unique fixed point $(y, z) \in L_{\mathbb{F}}^{2}\left(\Omega ; C\left(\left[\tau_{1, \varepsilon}, T\right]\right.\right.$; $H)) \times L_{\mathbb{F}}^{2}\left(\tau_{1, \varepsilon}, T ; H\right)$, which solves $(\underline{3.3})$ in $\left[\tau_{1, \varepsilon}, T\right]$ (in the sense of mild solution). Inductively, we conclude that (3.3) admits a mild solution $(y, z)$ in $\left[\tau_{k, \varepsilon}, \tau_{k-1, \varepsilon}\right]$ for $k=2, \cdots, N$. Furthermore,

$$
\begin{aligned}
& |z|_{L_{\mathbb{F}}^{2}\left(\tau_{1, \varepsilon}, T ; H\right)}^{2} \\
\leq & \mathbb{E} \int_{\tau_{1, \varepsilon}}^{T}\left|e^{A^{*}(T-s)} l(s)+\int_{s}^{T} e^{A^{*}(r-s)} \kappa(r, s) d r\right|_{H}^{2} d s \\
\leq & 4 \mathbb{E} \int_{\tau_{1, \varepsilon}}^{T}\left|e^{A^{*}(T-s)} l(s)\right|_{H}^{2} d s+\frac{3}{2} \mathbb{E} \int_{\tau_{1, \varepsilon}}^{T}\left|\int_{s}^{T} e^{A^{*}(r-s)} \kappa(r, s) d r\right|_{H}^{2} d s \\
\leq & \mathcal{C}|\xi|_{L_{\mathcal{F}_{T}}^{2}(\Omega ; H)}^{2}+\frac{3}{2} \widetilde{M_{T}}\left\{\int_{\tau_{1, \varepsilon}}^{T}\left[\mathbb{E}\left|A_{1}(s)^{*} y(s)+C(s)^{*} z(s)+h(s)\right|_{H}^{2}\right]^{\frac{1}{2}} d s\right\}^{2} \\
\leq & \mathcal{C}|\xi|_{L_{\mathcal{F}_{T}}^{2}(\Omega ; H)}^{2}+\mathcal{C}|h|_{L_{\mathbb{F}}^{2}\left(\Omega ; L^{1}\left(\tau_{1, \varepsilon}, T ; H\right)\right)}^{2}+2 \widetilde{M_{T}^{2}} \varepsilon\left(|y|_{L_{\mathbb{F}}^{2}\left(\Omega ; C\left(\left[\tau_{1, \varepsilon}, T\right] ; H\right)\right)}^{2}+|z|_{L_{\mathbb{F}}^{2}\left(\tau_{1, \varepsilon}, T ; H\right)}^{2}\right)
\end{aligned}
$$

and

$$
\begin{aligned}
& |y|_{L_{\mathbb{F}}^{2}\left(\Omega ; C\left(\left[\tau_{1, \varepsilon}, T\right] ; H\right)\right)}^{2} \\
\leq & \mathbb{E}\left[\sup _{t \in\left[\tau_{1, \varepsilon}, T\right]}\left|e^{A^{*}(T-t)} \xi-\int_{t}^{T} e^{A^{*}(s-t)}\left(A_{1}^{*} \tilde{y}+C^{*} \tilde{z}+h\right) d s-\int_{t}^{T} e^{A^{*}(s-t)} z(s) d W(s)\right|_{H}\right]^{2} \\
\leq & \mathcal{C}|\xi|_{L_{\mathcal{F}_{T}}^{2}(\Omega ; H)}^{2}+2 \mathbb{E}\left(\sup _{t \in\left[\tau_{1, \varepsilon}, T\right]}\left|\int_{t}^{T} e^{A^{*}(s-t)}\left(A_{1}^{*} \tilde{y}+C^{*} \tilde{z}\right) d r\right|_{H}^{2}+2 \mathbb{E} \int_{\tau_{1, \varepsilon}}^{T}\left|e^{A^{*}(s-t)} z\right|_{H}^{2} d s\right. \\
& +\mathcal{C}|h|_{L_{\mathbb{F}}^{2}\left(\Omega ; L^{1}(0, T ; H)\right)}^{2} \\
\leq & \mathcal{C}|\xi|_{L_{\mathcal{F}_{T}}^{2}(\Omega ; H)}^{2}+\mathcal{C}|h|_{L_{\mathbb{F}}^{1}\left(0, T ; L^{2}(\Omega ; H)\right)}^{2}+4 \widetilde{M}_{T}^{2}|y|_{L_{\mathbb{F}}^{2}\left(\Omega ; C\left(\left[\tau_{1, \varepsilon}, T\right] ; H\right)\right)}^{2}+6 \widetilde{M}_{T}^{2}|z|_{L_{\mathbb{F}}^{2}\left(\tau_{1, \varepsilon}, T ; H\right)}^{2} .
\end{aligned}
$$

It follows from (7.22), (7.23) and the choice of $\tau_{1, \varepsilon}$ that

$$
|(y, z)|_{L_{\mathbb{F}}^{2}\left(\Omega ; C\left(\left[\tau_{1, \varepsilon}, T\right] ; H\right)\right) \times L_{\mathbb{F}}^{2}\left(\tau_{1, \varepsilon}, T ; H\right)}^{2} \leq \mathcal{C}\left(|\xi|_{L_{\mathcal{F}_{T}}^{2}(\Omega ; H)}^{2}+|h|_{L_{\mathbb{F}}^{1}\left(0, T ; L^{2}(\Omega ; H)\right)}^{2}\right) .
$$

Repeating the above argument, we obtain (3.4). The uniqueness of the solution is obvious. 


\subsection{Proof of Lemma 3.3}

Proof of Lemma 3.3: By Lemmas 3.1 and 3.2, the equation (3.5) admits a mild solution $(\bar{x}(\cdot), \bar{y}(\cdot), \bar{z}(\cdot))$. Since $\bar{\Theta}(\cdot)$ is an optimal feedback operator of Problem (SLQ), $(\bar{x}, \Theta \bar{x})$ is an optimal pair. Put $\bar{u}(\cdot)=\Theta \bar{x}(\cdot)$. Fix arbitrarily a control $u(\cdot) \in L_{\mathbb{F}}^{2}(t, T ; U)$ and put

$$
u^{\varepsilon}(\cdot)=\bar{u}(\cdot)+\varepsilon[u(\cdot)-\bar{u}(\cdot)]=(1-\varepsilon) \bar{u}(\cdot)+\varepsilon u(\cdot) \in L_{\mathbb{F}}^{2}(t, T ; U), \quad \forall \varepsilon \in[0,1] .
$$

Denote by $x^{\varepsilon}(\cdot)$ the solution to (1.1) corresponding to the control $u^{\varepsilon}(\cdot)$. Write $x_{1}^{\varepsilon}(\cdot)=$ $\frac{1}{\varepsilon}\left[x^{\varepsilon}(\cdot)-\bar{x}(\cdot)\right]$ and $\delta u(\cdot)=u(\cdot)-\bar{u}(\cdot)$. It is easy to see that $x_{1}^{\varepsilon}(\cdot)$ solves the following SEE:

$$
\left\{\begin{array}{l}
d x_{1}^{\varepsilon}=\left[\left(A+A_{1}\right) x_{1}^{\varepsilon}+B \delta u\right] d s+\left(C x_{1}^{\varepsilon}+D \delta u\right) d W(s) \quad \text { in }(t, T], \\
x_{1}^{\varepsilon}(t)=0 .
\end{array}\right.
$$

Thanks to that $(\bar{x}(\cdot), \bar{u}(\cdot))$ is an optimal pair of Problem (SLQ), it holds that

$$
\begin{aligned}
0 & \leq \lim _{\varepsilon \rightarrow 0} \frac{\mathcal{J}\left(t, \eta ; u^{\varepsilon}(\cdot)\right)-\mathcal{J}(t, \eta ; \bar{u}(\cdot))}{\varepsilon} \\
& =\mathbb{E} \int_{t}^{T}\left(\left\langle Q \bar{x}, x_{1}^{\varepsilon}\right\rangle+\langle R \bar{u}, \delta u\rangle\right) d s+\mathbb{E}\left\langle G \bar{x}(T), x_{1}^{\varepsilon}(T)\right\rangle .
\end{aligned}
$$

By Itô's formula,

$$
\mathbb{E}\left\langle G \bar{x}(T), x_{1}^{\varepsilon}(T)\right\rangle+\mathbb{E} \int_{t}^{T}\left\langle Q \bar{x}, x_{1}^{\varepsilon}\right\rangle d s=\mathbb{E} \int_{t}^{T}(\langle B \delta u, \bar{y}\rangle+\langle D \delta u, \bar{z}\rangle) d s .
$$

Combining (7.26) and (7.27), we find that for any $u(\cdot) \in L_{\mathbb{F}}^{2}(0, T ; U)$,

$$
\mathbb{E} \int_{t}^{T}\left\langle R \bar{u}+B^{*} \bar{y}+D^{*} \bar{z}, u-\bar{u}\right\rangle d s \geq 0
$$

This implies (3.6).

\subsection{Proof of Lemma 3.4}

Proof of Lemma 3.4: Without loss of generality, we assume that $t=0$.

In the beginning, we prove the first equality in (3.16). Let $\varepsilon \in[0, T]$ such that

$$
\varepsilon=\sup \left\{r \in[0, T]: 10 M_{T}^{2}\left[\left(\int_{0}^{r}\left|A_{1}\right|_{\mathcal{L}(H)} d s\right)^{2}+\int_{0}^{r}|C|_{\mathcal{L}(H)}^{2} d s\right] \leq \frac{1}{2}\right\} \wedge T .
$$

For any $r \in[0, \varepsilon]$, it follows from (3.5) and (3.14) that

$$
\begin{aligned}
& \sup _{r \in[0, \varepsilon]} \mathbb{E}\left(\left|x(r)-x_{n}(r)\right|_{H}\right)^{2} \\
\leq & 5\left(\sup _{r \in[0, \varepsilon]} \mathbb{E}\left|\left(e^{A t}-e^{A t} \Gamma_{n}\right) \zeta\right|_{H}^{2}+\sup _{r \in[0, \varepsilon]} \mathbb{E}\left(\left|\int_{0}^{r}\left(e^{A(r-s)} A_{1} x-e^{A(r-s)} \Gamma_{n} A_{1, n} x_{n}\right) d s\right|_{H}\right)^{2}\right.
\end{aligned}
$$




$$
\begin{aligned}
& +\sup _{r \in[0, \varepsilon]} \mathbb{E}\left(\left|\int_{0}^{r}\left(e^{A(r-s)} B u-e^{A(r-s)} \Gamma_{n} B_{n} u\right) d s\right|_{H}\right)^{2}+\int_{0}^{\varepsilon}\left|e^{A(r-s)} C x-e^{A(r-s)} \Gamma_{n} C_{n} x_{n}\right|_{H}^{2} d s \\
& \left.+\int_{0}^{\varepsilon}\left|e^{A(r-s)} D u-e^{A(r-s)} \Gamma_{n} D_{n} u\right|_{H}^{2} d s\right) .
\end{aligned}
$$

Let us estimate the terms in the right hand side of (7.30). Clearly,

$$
\begin{aligned}
& \sup _{r \in[0, \varepsilon]} \mathbb{E}\left[\left|\int_{0}^{r}\left(e^{A(r-s)} A_{1} x-e^{A(r-s)} \Gamma_{n} A_{1, n} x_{n}\right) d s\right|_{H}\right]^{2} \\
& \leq \mathcal{C} \mathbb{E}\left(\int_{0}^{\varepsilon}\left|\left(A_{1}-\Gamma_{n} A_{1} \Gamma_{n}\right) x\right|_{H} d s\right)^{2}+2 M_{T}^{2}\left(\int_{0}^{\varepsilon}\left|A_{1}\right|_{\mathcal{L}(H)} d s\right)^{2} \sup _{r \in[0, \varepsilon]} \mathbb{E}\left|x(r)-x_{n}(r)\right|_{H}^{2}, \\
& \sup _{r \in[0, \varepsilon]} \mathbb{E}\left|\int_{0}^{r}\left[e^{A(r-s)} B u-e^{A(r-s)} \Gamma_{n} B_{n} u\right] d s\right|_{H}^{2} \leq \mathcal{C}\left(\int_{0}^{\varepsilon}\left|\left(B-B_{n}\right) u\right|_{H} d s\right)^{2}, \\
& \quad \mathbb{E} \int_{0}^{\varepsilon}\left|e^{A(r-s)} C x-e^{A(r-s)} \Gamma_{n} C_{n} x_{n}\right|_{H}^{2} d s \\
& \leq \mathcal{C E} \int_{0}^{\varepsilon}\left|\left(C-C_{n}\right) x\right|_{H}^{2} d s+2 M_{T}^{2}\left(\int_{0}^{\varepsilon}|C|_{\mathcal{L}(H)}^{2} d s\right) \sup _{r \in[0, \varepsilon]} \mathbb{E}\left|x(r)-x_{n}(r)\right|_{H}^{2},
\end{aligned}
$$

and

$$
\mathbb{E} \int_{0}^{\varepsilon}\left|e^{A(r-s)} D u-e^{A(r-s)} \Gamma_{n} D_{n} u\right|_{H}^{2} d s \leq \mathcal{C} \int_{0}^{\varepsilon}\left|\left(D-D_{n}\right) u\right|_{H}^{2} d s .
$$

Combining (7.30), (17.32), (7.33) and (7.34), and noting (7.29), we get that

$$
\begin{aligned}
\sup _{r \in[0, \varepsilon]} \mathbb{E}\left|x(r)-x_{n}(r)\right|_{H}^{2} \\
\leq \mathcal{C} \mathbb{E}\left\{\left|\left(e^{A r}-e^{A r} \Gamma_{n}\right) \zeta\right|_{H}^{2}+\left[\int_{0}^{\varepsilon}\left|\left(A_{1}-\Gamma_{n} A_{1} \Gamma_{n}\right) x\right|_{H} d s\right]^{2}\right. \\
\left.+\left[\int_{0}^{\varepsilon}\left|\left(B-B_{n}\right) u\right|_{H} d s\right]^{2}+\int_{0}^{\varepsilon}\left|\left(C-C_{n}\right) x\right|_{H}^{2} d s+\int_{0}^{\varepsilon}\left|\left(D-D_{n}\right) u\right|_{H}^{2} d s\right\} .
\end{aligned}
$$

From (3.10), we know that

$$
\lim _{n \rightarrow \infty}\left|\left(e^{A r}-e^{A r} \Gamma_{n}\right) \zeta\right|_{H}^{2}=0
$$

Since

$$
\left[\int_{0}^{\varepsilon}\left|\left(A_{1}-\Gamma_{n} A_{1} \Gamma_{n}\right) x\right|_{H} d s\right]^{2} \leq\left[\int_{0}^{\varepsilon}\left|A_{1}\right|_{\mathcal{L}(H)}|x|_{H} d s\right]^{2}
$$

we get from Lebesgue's dominated convergence theorem that

$$
\lim _{n \rightarrow+\infty} \mathbb{E}\left[\int_{0}^{\varepsilon}\left|\left(A_{1}-\Gamma_{n} A_{1} \Gamma_{n}\right) x\right|_{H} d s\right]^{2}=\mathbb{E} \lim _{n \rightarrow+\infty}\left[\int_{0}^{\varepsilon}\left|\left(A_{1}-\Gamma_{n} A_{1} \Gamma_{n}\right) x\right|_{H} d s\right]^{2}
$$

Noting that

$$
\left|e^{A(r-s)}\left(A_{1}-\Gamma_{n} A_{1} \Gamma_{n}\right) x\right|_{H} \leq M_{T}\left|A_{1}\right|_{\mathcal{L}(H)}|x|_{H},
$$

by Lebesgue's dominated convergence theorem, (7.37) and (3.12), we have that

$$
\lim _{n \rightarrow+\infty} \mathbb{E}\left[\int_{0}^{\varepsilon}\left|\left(A_{1}-\Gamma_{n} A_{1} \Gamma_{n}\right) x\right|_{H} d s\right]^{2}=\mathbb{E}\left[\int_{0}^{\varepsilon} \lim _{n \rightarrow+\infty}\left|\left(A_{1}-\Gamma_{n} A_{1} \Gamma_{n}\right) x\right|_{H} d s\right]^{2}=0 .
$$


By a similar argument, we can prove that

$$
\lim _{n \rightarrow+\infty} \mathbb{E}\left\{\left[\int_{0}^{\varepsilon}\left|\left(B-B_{n}\right) u\right|_{H} d s\right]^{2}+\int_{0}^{\varepsilon}\left|\left(C-C_{n}\right) x\right|_{H}^{2} d s+\int_{0}^{\varepsilon}\left|\left(D-D_{n}\right) u\right|_{H}^{2} d s\right\}=0 .
$$

It follows from (7.35), (7.38) and (7.39) that

$$
\lim _{n \rightarrow+\infty} \sup _{r \in[0, \varepsilon]} \mathbb{E}\left|x(r)-x_{n}(r)\right|_{H}^{2}=0
$$

Repeating the above argument yields the first equality in (3.16). The proof for the fourth one is similar. Now we consider the second and third one.

Let

$$
T_{1} \triangleq \inf \left\{r \in[0, T]:\left[24\left(14 \widetilde{M}_{T}^{2}+1\right) \widetilde{M}_{T}^{2}+14 \widetilde{M}_{T}^{2}\right]\left[\left(\int_{r}^{T}\left|A_{1}^{*}\right|_{\mathcal{L}(H)} d s\right)^{2}+\int_{r}^{T}\left|C^{*}\right|_{\mathcal{L}(H)}^{2} d s\right] \leq \frac{1}{2}\right\} .
$$

We first recall that

$$
y(r)=e^{A^{*}(T-r)} G x(T)-\int_{r}^{T} e^{A^{*}(s-r)}\left(A_{1}^{*} y+C^{*} z+Q x\right) d s-\int_{r}^{T} e^{A^{*}(s-r)} z(s) d W(s),
$$

and

$$
z(r)=e^{A^{*}(T-r)} l(r)+\int_{r}^{T} e^{A^{*}(\sigma-r)} \kappa(\sigma, r) d \sigma
$$

where $l(\cdot) \in L_{\mathbb{F}}^{2}(0, T ; H)$ such that

$$
\mathbb{E}\left(G x(T) \mid \mathcal{F}_{r}\right)=G \mathbb{E} x(T)+\int_{0}^{r} l(\sigma) d W(\sigma)
$$

and $\kappa(\cdot, \cdot) \in L^{1}\left(0, T ; L_{\mathbb{F}}^{2}(0, T ; H)\right)$ such that for a.e. $s \in[0, T]$,

$$
\left(A_{1}^{*} y+C^{*} z+Q x\right)(s)=\mathbb{E}\left(A_{1}^{*} y+C^{*} z+Q x\right)(s)+\int_{0}^{s} \kappa(s, \sigma) d W(\sigma)
$$

and

$$
|\kappa(\cdot, \cdot)|_{L^{1}\left(r, T ; L_{\mathbb{F}}^{2}(r, T ; H)\right)} \leq\left|A_{1}^{*} y+C^{*} z+Q x\right|_{L_{\mathbb{F}}^{1}\left(r, T ; L^{2}(\Omega ; H)\right)} \cdot
$$

Furthermore,

$$
\begin{aligned}
y_{n}(r)= & e^{A_{n}^{*}(T-r)} G_{n} x_{n}(T)-\int_{r}^{T} e^{A_{n}^{*}(s-r)}\left(A_{1, n}^{*} y_{n}+C_{n}^{*} z_{n}+Q_{n} x_{n}\right) d s \\
& -\int_{r}^{T} e^{A_{n}^{*}(s-r)} z_{n}(s) d W(s),
\end{aligned}
$$

and

$$
z_{n}(r)=e^{A_{n}^{*}(T-r)} l_{n}(r)+\int_{r}^{T} e^{A_{n}^{*}(\sigma-r)} \kappa_{n}(\sigma, r) d \sigma,
$$

where $l_{n}(\cdot) \in L_{\mathbb{F}}^{2}(0, T ; H)$ such that

$$
\mathbb{E}\left(G_{n} x_{n}(T) \mid \mathcal{F}_{r}\right)=G_{n} \mathbb{E} x_{n}(T)+\int_{0}^{r} l_{n}(\sigma) d W(\sigma) .
$$


and $\kappa_{n}(\cdot, \cdot) \in L^{1}\left(0, T ; L_{\mathbb{F}}^{2}(0, T ; H)\right)$ such that for a.e. $s \in[0, T]$,

$$
\left(A_{1, n}^{*} y_{n}+C_{n}^{*} z_{n}+Q_{n} x_{n}\right)(s)=\mathbb{E}\left(A_{1, n}^{*} y_{n}+C_{n}^{*} z_{n}+Q_{n} x_{n}\right)(s)+\int_{0}^{s} \kappa_{n}(s, \sigma) d W(\sigma)
$$

and

$$
\left|\kappa_{n}(\cdot, \cdot)\right|_{L^{1}\left(r, T ; L_{\mathbb{F}}^{2}(r, T ; H)\right)} \leq\left|A_{1, n}^{*} y_{n}+C_{n}^{*} z_{n}+Q_{n} x_{n}\right|_{L_{\mathbb{F}}^{1}\left(r, T ; L^{2}(\Omega ; H)\right)} \cdot
$$

Then,

$$
\begin{aligned}
\mathbb{E} \sup _{r \in\left[T_{1}, T\right]}\left|y(r)-y_{n}(r)\right|_{H}^{2}+\left(14 \widetilde{M}_{T}^{2}+1\right) \mathbb{E} \int_{T_{1}}^{T}\left|z(r)-z_{n}(r)\right|_{H}^{2} d r \\
\leq \mathcal{C E} \sup _{r \in\left[T_{1}, T\right]}\left[\left|\left(e^{A^{*}(T-r)}-e^{A_{n}^{*}(T-r)}\right) G x(T)\right|_{H}^{2}+\left|e^{A_{n}^{*}(T-r)}\left(G-G_{n}\right) x(T)\right|_{H}^{2}\right. \\
+\left|e^{A_{n}^{*}(T-r)} G_{n}\left(x(T)-x_{n}(T)\right)\right|_{H}^{2}+\left(\int_{r}^{T}\left|\left(e^{A^{*}(s-r)}-e^{A_{n}^{*}(s-r)}\right) A_{1}^{*} y\right|_{H} d s\right)^{2} \\
+\left(\int_{r}^{T}\left|e^{A_{n}^{*}(s-r)}\left(A_{1}^{*}-A_{1, n}^{*}\right) y\right|_{H} d s\right)^{2}+\left(\int_{r}^{T}\left|e^{A_{n}^{*}(s-r)} A_{1, n}^{*}\left(y-y_{n}\right)\right|_{H} d s\right)^{2} \\
+\left(\int_{r}^{T}\left|\left(e^{A^{*}(s-r)}-e^{A_{n}^{*}(s-r)}\right) C^{*} z\right|_{H} d s\right)^{2}+\left(\int_{r}^{T}\left|e^{A_{n}^{*}(s-r)}\left(C^{*}-C_{n}^{*}\right) z\right|_{H} d s\right)^{2}(7.51) \\
+\left(\int_{r}^{T}\left|e^{A_{n}^{*}(s-r)} C_{n}^{*}\left(z-z_{n}\right)\right|_{H} d s\right)^{2}+\mathbb{E}\left(\int_{r}^{T}\left|\left(e^{A^{*}(s-r)}-e^{A_{n}^{*}(s-r)}\right) Q^{*} x\right|_{H} d s\right)^{2} \\
+\left(\int_{r}^{T}\left|e^{A_{n}^{*}(s-r)}\left(Q^{*}-Q_{n}^{*}\right) x\right|_{H} d s\right)^{2}+\left(\int_{r}^{T}\left|e^{A_{n}^{*}(s-r)} Q_{n}^{*}\left(x-x_{n}\right)\right|_{H} d s\right)^{2} \\
\left.+\int_{r}^{T}\left|\left(e^{A^{*}(s-r)}-e^{A_{n}^{*}(s-r)}\right) z(s)\right|_{H}^{2} d s+\int_{r}^{T}\left|e^{A_{n}^{*}(s-r)}\left(z(s)-z_{n}(s)\right)\right|_{H}^{2} d s\right] \\
+4\left(14 \widetilde{M}_{T}^{2}+1\right) \mathbb{E}\left[\int_{T_{1}}^{T}\left|\left(e^{A^{*}(T-s)}-e^{A_{n}^{*}(T-s)}\right) l(s)\right|_{H}^{2} d s+\int_{T_{1}}^{T}\left|e^{A_{n}^{*}(T-s)}\left(l(s)-l_{n}(s)\right)\right|_{H}^{2} d s\right. \\
+\int_{T_{1}}^{T}\left|\int_{s}^{T}\left(e^{A^{*}(\sigma-s)}-e^{A_{n}^{*}(\sigma-s)}\right) \kappa(\sigma, s) d \sigma\right|_{H}^{2} d s \\
\left.+\int_{T_{1}}^{T}\left|\int_{s}^{T} e^{A_{n}^{*}(\sigma-s)}\left(\kappa(\sigma, s)-\kappa_{n}(\sigma, s)\right) d \sigma\right|_{H}^{2} d s\right] .
\end{aligned}
$$

Since

$$
\left|\left(e^{A^{*}(T-r)}-e^{A_{n}^{*}(T-r)}\right) G x(T)\right|_{H}^{2} \leq \mathcal{C}|x(T)|_{H}^{2},
$$

it follows from (3.11) and Lebesgue's dominated convergence theorem that

$$
\lim _{n \rightarrow \infty} \mathbb{E} \sup _{r \in\left[T_{1}, T\right]}\left|\left(e^{A^{*}(T-r)}-e^{A_{n}^{*}(T-r)}\right) G x(T)\right|_{H}^{2}=0 .
$$

Similarly, we have that

$$
\lim _{n \rightarrow \infty} \mathbb{E} \sup _{r \in\left[T_{1}, T\right]}\left|e^{A_{n}^{*}(T-r)}\left(G-G_{n}\right) x(T)\right|_{H}^{2}=0 .
$$


Similar to the proof of (7.38), we can obtain that

$$
\begin{aligned}
\lim _{n \rightarrow \infty} \mathbb{E} \sup _{r \in\left[T_{1}, T\right]}\left\{\left[\int_{r}^{T}\left|\left(e^{A^{*}(s-r)}-e^{A_{n}^{*}(s-r)}\right) A_{1}^{*} y\right|_{H} d s\right]^{2}+\left[\int_{r}^{T}\left|e^{A_{n}^{*}(s-r)}\left(A_{1}^{*}-A_{1, n}^{*}\right) y\right|_{H} d s\right]^{2}\right. \\
+\left[\int_{r}^{T}\left|\left(e^{A^{*}(s-r)}-e^{A_{n}^{*}(s-r)}\right) C^{*} z\right|_{H} d s\right]^{2}+\left[\int_{r}^{T}\left|e^{A_{n}^{*}(s-r)}\left(C^{*}-C_{n}^{*}\right) z\right|_{H} d s\right]^{2} \\
+\left[\int_{r}^{T}\left|\left(e^{A^{*}(s-r)}-e^{A_{n}^{*}(s-r)}\right) Q^{*} x\right|_{H} d s\right]^{2}+\left[\int_{r}^{T}\left|e^{A_{n}^{*}(s-r)}\left(Q^{*}-Q_{n}^{*}\right) x\right|_{H} d s\right]^{2}(7.54) \\
+\int_{r}^{T}\left|\left(e^{A^{*}(s-r)}-e^{A_{n}^{*}(s-r)}\right) z\right|_{H}^{2} d s+\int_{T_{1}}^{T}\left|\left(e^{A^{*}(T-s)}-e^{A_{n}^{*}(T-s)}\right) l(s)\right|_{H}^{2} d s \\
\left.+\int_{T_{1}}^{T}\left|\int_{s}^{T}\left(e^{A^{*}(\sigma-s)}-e^{A_{n}^{*}(\sigma-s)}\right) \kappa(\sigma, s) d \sigma\right|_{H}^{2} d s\right\}=0 .
\end{aligned}
$$

By the first equality in (3.16), we get that

$$
\begin{aligned}
& \lim _{n \rightarrow \infty} \mathbb{E} \sup _{r \in\left[T_{1}, T\right]}\left[\left|e^{A_{n}^{*}(T-r)} G_{n}\left(x(T)-x_{n}(T)\right)\right|_{H}^{2}+\left(\int_{r}^{T}\left|e^{A_{n}^{*}(s-r)} Q_{n}^{*}\left(x-x_{n}\right)\right|_{H} d s\right)^{2}\right] \\
& \leq \mathcal{C} \lim _{n \rightarrow \infty}\left[\mathbb{E}\left|x(T)-x_{n}(T)\right|_{H}^{2}+\left(\int_{T_{1}}^{T}\left|Q^{*}\right|_{\mathcal{L}(H)} d s\right)^{2} \sup _{r \in\left[T_{1}, T\right]} \mathbb{E}\left|x(r)-x_{n}(r)\right|_{H}\right]=0 .
\end{aligned}
$$

Clearly,

$$
\begin{aligned}
& \mathbb{E} \sup _{r \in\left[T_{1}, T\right]}\left(\int_{r}^{T}\left|e^{A_{n}^{*}(s-r)} A_{1, n}^{*}\left(y-y_{n}\right)\right|_{H} d s\right)^{2}+\mathbb{E} \sup _{r \in\left[T_{1}, T\right]}\left(\int_{r}^{T}\left|e^{A_{n}^{*}(s-r)} C_{n}^{*}\left(z-z_{n}\right)\right|_{H} d s\right)^{2} \\
& \leq \widetilde{M_{T}^{2}}\left(\int_{T_{1}}^{T}\left|A_{1}^{*}\right|_{\mathcal{L}(H)} d s\right)^{2} \mathbb{E} \sup _{r \in\left[T_{1}, T\right]}\left|y(r)-y_{n}(r)\right|_{H}^{2}+\widetilde{M_{T}^{2}}\left(\int_{T_{1}}^{T}\left|C^{*}\right|_{\mathcal{L}(H)}^{2} d s\right) \mathbb{E} \int_{T_{1}}^{T}\left|z(r)-z_{n}(r)\right|_{H}^{2} d s .
\end{aligned}
$$

From (7.43) and (7.48), we have that

$$
\begin{aligned}
& \mathbb{E} \int_{T_{1}}^{T}\left|e^{A_{n}^{*}(T-s)}\left(l(s)-l_{n}(s)\right)\right|_{H}^{2} d s \leq \widetilde{M}_{T}^{2} \mathbb{E} \int_{T_{1}}^{T}\left|\left(l(s)-l_{n}(s)\right)\right|_{H}^{2} d s \\
& \leq \widetilde{M}_{T}^{2} \mathbb{E}\left|G x(T)-\mathbb{E}\left(G x(T) \mid \mathcal{F}_{T_{1}}\right)-G_{n} x_{n}(T)+\mathbb{E}\left(G_{n} x_{n}(T) \mid \mathcal{F}_{T_{1}}\right)\right|_{H}^{2} \\
& \leq 2 \widetilde{M_{T}^{2}} \mathbb{E}\left|G x(T)-G_{n} x_{n}(T)\right|_{H}^{2}+2 \widetilde{M}_{T}^{2} \mathbb{E}\left|\mathbb{E}\left(G x(T) \mid \mathcal{F}_{T_{1}}\right)-\mathbb{E}\left(G_{n} x_{n}(T) \mid \mathcal{F}_{T_{1}}\right)\right|_{H}^{2} \\
& \leq 4 \widetilde{M_{T}^{2}} \mathbb{E}\left|G x(T)-G_{n} x_{n}(T)\right|_{H}^{2} \leq 8 \widetilde{M}_{T}^{2} \mathbb{E}\left|\left(G-G_{n}\right) x(T)\right|_{H}^{2}+\mathcal{C} \widetilde{M}_{T}^{2} \mathbb{E}\left|x(T)-x_{n}(T)\right|_{H}^{2}
\end{aligned}
$$

Since

$$
\left|\left(G-G_{n}\right) x(T)\right|_{H}^{2} \leq \mathcal{C}|x(T)|_{H}^{2}
$$

it follows from Lebesgue's dominated convergence theorem and (3.12) that

$$
\lim _{n \rightarrow \infty} \mathbb{E}\left|\left(G-G_{n}\right) x(T)\right|_{H}^{2}=\mathbb{E} \lim _{n \rightarrow \infty}\left|\left(G-G_{n}\right) x(T)\right|_{H}^{2}=0
$$

By the first equality in (3.16) again, we get that

$$
\lim _{n \rightarrow \infty} \mathbb{E}\left|x(T)-x_{n}(T)\right|_{H}^{2}=0 .
$$


This, together with (7.57) and (7.58), implies that

$$
\lim _{n \rightarrow \infty} \mathbb{E} \int_{T_{1}}^{T}\left|e^{A_{n}^{*}(T-s)}\left(l(s)-l_{n}(s)\right)\right|_{H}^{2} d s=0 .
$$

From (7.44), (17.45), (7.49) and (17.50), we conclude that

$$
\begin{aligned}
& 4\left(14 \widetilde{M}_{T}^{2}+1\right) \mathbb{E} \int_{T_{1}}^{T}\left|\int_{s}^{T} e^{A_{n}^{*}(\sigma-s)}\left(\kappa(\sigma, s)-\kappa_{n}(\sigma, s)\right) d \sigma\right|_{H}^{2} d s \\
\leq & 4\left(14 \widetilde{M_{T}^{2}}+1\right) \widetilde{M_{T}^{2}}\left[\int_{T_{1}}^{T}\left(\mathbb{E}\left|A_{1}^{*} y+C^{*} z+Q x-A_{1, n}^{*} y_{n}-C_{n}^{*} z_{n}-Q_{n} x_{n}\right|_{H}^{2}\right)^{\frac{1}{2}} d s\right]^{2} \\
\leq & 24\left(14 \widetilde{M}_{T}^{2}+1\right) \widetilde{M_{T}^{2}}\left\{\left[\int_{T_{1}}^{T}\left(\mathbb{E}\left|\left(A_{1}^{*}-A_{1, n}^{*}\right) y\right|_{H}^{2}\right)^{\frac{1}{2}} d s\right]^{2}+\left(\int_{T_{1}}^{T}\left|A_{1, n}^{*}\right| \mathcal{L}(H) d s\right)^{2} \sup _{r \in\left[T_{1}, T\right]}\left|y(r)-y_{n}(r)\right|_{H}^{2}\right. \\
& +\left[\int_{T_{1}}^{T}\left(\mathbb{E}\left|\left(C^{*}-C_{n}^{*}\right) z\right|_{H}^{2}\right)^{\frac{1}{2}} d s\right]^{2}+\left(\int_{T_{1}}^{T}\left|C_{n}^{*}\right|_{\mathcal{L}(H)}^{2} d s\right) \mathbb{E} \int_{T_{1}}^{T}\left|z-z_{n}\right|_{H}^{2} d s \\
& \left.+\left[\int_{T_{1}}^{T}\left(\mathbb{E}\left|\left(Q^{*}-Q_{n}^{*}\right) x\right|_{H}^{2}\right)^{\frac{1}{2}} d s\right]^{2}+\left(\int_{T_{1}}^{T}\left|Q_{n}^{*}\right|_{\mathcal{L}(H)} d s\right)^{2} \sup _{r \in\left[T_{1}, T\right]}\left|x(r)-x_{n}(r)\right|_{H}^{2}\right\} .
\end{aligned}
$$

Combining (7.40), (77.52), (7.53), (7.55), (7.54), (7.56), (7.59) and (7.60), we find that

$$
\lim _{n \rightarrow \infty}\left(\mathbb{E} \sup _{r \in\left[T_{1}, T\right]}\left|y(r)-y_{n}(r)\right|_{H}^{2}+\mathbb{E} \int_{T_{1}}^{T}\left|z(r)-z_{n}(r)\right|_{H}^{2} d r\right)=0 .
$$

By repeating the above argument, we obtain the second and third equality in (3.16).

\subsection{Proof of Propositions 3.13 .3}

Proof of Proposition 3.1]: Let $\tau \in[0, T)$ such that

$$
\int_{\tau}^{T}\left(2\left|A_{1}+B \Theta\right|_{\mathcal{L}(H)}+|C+D \Theta|_{\mathcal{L}(H)}^{2}\right) d s<\frac{1}{2 M_{T}^{2}}
$$

Define a map $\mathcal{G}: C_{\mathcal{S}}([\tau, T] ; \mathcal{L}(H)) \rightarrow C_{\mathcal{S}}([\tau, T] ; \mathcal{L}(H))$ as follows:

$$
\begin{aligned}
\mathcal{G}(P)(r) \zeta= & e^{(T-r) A^{*}} G e^{(T-r) A} \eta+\int_{r}^{T} e^{(s-r) A^{*}}\left[P\left(A_{1}+B \Theta\right)+\left(A_{1}+B \Theta\right)^{*} P\right. \\
& \left.+(C+D \Theta)^{*} P(C+D \Theta)+\Theta^{*} R \Theta+Q\right] e^{(s-r) A} \zeta d s, \quad \forall \zeta \in H .
\end{aligned}
$$

Let $P_{1}, P_{2} \in C_{\mathcal{S}}([0, T] ; \mathcal{L}(H))$. Then, for each $\zeta \in H$,

$$
\begin{aligned}
\sup _{r \in[\tau, T]}\left|\left(\mathcal{G}\left(P_{1}\right)-\mathcal{G}\left(P_{1}\right)\right)(r) \zeta\right|_{H} & \\
=\sup _{r \in[\tau, T]} \mid & \int_{r}^{T} e^{(s-r) A^{*}}\left[\left(P_{1}-P_{2}\right)\left(A_{1}+B \Theta\right)+\left(A_{1}+B \Theta\right)^{*}\left(P_{1}-P_{2}\right)\right. \\
& \left.\quad+(C+D \Theta)^{*}\left(P_{1}-P_{2}\right)(C+D \Theta)\right]\left.e^{(s-r) A} \zeta d s\right|_{H}
\end{aligned}
$$




$$
\begin{aligned}
& \leq \int_{\tau}^{T}\left|e^{(s-r) A^{*}}\right|_{\mathcal{L}(H)}\left[\left(\left|A_{1}+B \Theta\right|_{\mathcal{L}(H)}+\left|\left(A_{1}+B \Theta\right)^{*}\right|_{\mathcal{L}(H)}\right)\left|\left(P_{1}-P_{2}\right)\right|_{\mathcal{L}(H)}\right. \\
& \left.\quad+\left|(C+D \Theta)^{*}\right|_{\mathcal{L}(H)}\left|P_{1}-P_{2}\right|_{\mathcal{L}(H)}|(C+D \Theta)|_{\mathcal{L}(H)}\right]\left|e^{(s-r) A}\right|_{\mathcal{L}(H)}|\zeta|_{H} d s \\
& \leq M_{T}^{2} \int_{\tau}^{T}\left(2\left|A_{1}+B \Theta\right|_{\mathcal{L}(H)}+|C+D \Theta|_{\mathcal{L}(H)}^{2}\right) d s \sup _{r \in[\tau, T]}\left|\left(P_{1}-P_{2}\right)(r)\right|_{\mathcal{L}(H)}|\zeta|_{H} \\
& \leq \frac{1}{2} \sup _{r \in[\tau, T]}\left|\left(P_{1}-P_{2}\right)(r)\right|_{\mathcal{L}(H)}|\zeta|_{H} .
\end{aligned}
$$

Therefore,

$$
\sup _{r \in[\tau, T]}\left|\left(\mathcal{G}\left(P_{1}\right)-\mathcal{G}\left(P_{1}\right)\right)(r)\right|_{\mathcal{L}(H)} \leq \frac{1}{2} \sup _{r \in[\tau, T]}\left|\left(P_{1}-P_{2}\right)(r)\right|_{\mathcal{L}(H)} .
$$

This deduces that $\mathcal{G}$ is contractive. Consequently, there is a unique fixed point of $\mathcal{G}$, which is the mild solution to (3.17) on $[\tau, T]$. Repeating this process gives us the unique $P \in C_{\mathcal{S}}([0, T] ; \mathcal{L}(H))$ which satisfies (3.18). On the other hand, it is obvious that if $P \in C_{\mathcal{S}}([0, T] ; \mathcal{L}(H))$ satisfying (3.18), then $P^{*} \in C_{\mathcal{S}}([0, T] ; \mathcal{L}(H))$ does. Hence, we have $P=P^{*}$, that is, $P \in C_{\mathcal{S}}([0, T] ; \mathcal{S}(H))$.

The uniqueness of the solution is obvious.

From (3.18), we see that for any $\zeta \in H$,

$$
\begin{aligned}
& \sup _{r \in[0, T]}|P(r) \zeta|_{H} \\
& \leq \sup _{r \in[0, T]}\left|e^{(T-r) A}\right|_{\mathcal{L}(H)}^{2}|G|_{\mathcal{L}(H)}|\zeta|_{H}+\int_{0}^{T}\left|e^{(s-r) A}\right|_{\mathcal{L}(H)}^{2}\left[\left(2\left|A_{1}+B \Theta\right|_{\mathcal{L}(H)}\right.\right. \\
& \left.\left.\quad+|C+D \Theta|_{\mathcal{L}(H)}^{2}\right)|P|_{\mathcal{L}(H)}+|\Theta|_{\mathcal{L}(H ; U)}^{2}|R|_{\mathcal{L}(U)}+|Q|_{\mathcal{L}(H)}\right]|\zeta|_{H} d s .
\end{aligned}
$$

This, together with Gronwall's inequality, implies the desired result.

Proof of Proposition 3.2: For any $\eta, \zeta \in H$, we have that

$$
\begin{aligned}
\langle P(r) \eta, \zeta\rangle= & \left\langle G e^{(T-r) A} \eta, e^{(T-r) A} \zeta\right\rangle+\int_{r}^{T}\left\langle\left[ P\left(A_{1}+B \Theta\right)+\left(A_{1}+B \Theta\right)^{*} P\right.\right. \\
& \left.\left.+(C+D \Theta)^{*} P(C+D \Theta)+\Theta^{*} R \Theta+Q\right] e^{(s-r) A} \eta, e^{(s-r) A} \zeta\right\rangle d s
\end{aligned}
$$

If $\eta, \zeta \in D(A)$, it follows that $\langle P(r) \eta, \zeta\rangle$ is differentiable with respect to $r$. A simple computation implies (3.19).

The proof of Proposition 3.3 is similar to the one of Proposition 3.2, we omit it.

\subsection{Proof Proposition 3.4}

The proof of Proposition 3.4 is based on a standard argument involving a minimizing se-

quence and locally weak compactness of Hilbert spaces. We give it in the appendix for the completeness of the paper and the convenience for some readers. 
Proof Proposition 3.4: It follows from the uniform convexity of $u(\cdot) \mapsto \mathcal{J}(0,0 ; u(\cdot))$ that

$$
\mathcal{J}(0,0 ; u(\cdot)) \geq \lambda \mathbb{E} \int_{0}^{T}|u(s)|^{2} d s, \quad \forall u(\cdot) \in \mathcal{U}[0, T],
$$

for some $\lambda>0$. For any $t \in[0, T)$, and any $u(\cdot) \in \mathcal{U}[t, T]$, let

$$
v(s) \triangleq \begin{cases}0, & s \in[0, t), \\ u(s), & s \in[t, T] .\end{cases}
$$

Then $v(\cdot) \in \mathcal{U}[0, T]$. Since the initial state is 0 , the solution $x(\cdot)$ to

$$
\left\{\begin{array}{l}
d x=\left[\left(A+A_{1}\right) x+B v\right] d s+(C x+D v) d W(s) \quad \text { in }(0, T], \\
x(0)=0,
\end{array}\right.
$$

satisfies

$$
x(s)=0, \quad s \in[0, t] .
$$

Therefore,

$$
\mathcal{J}(t, 0 ; u(\cdot))=\mathcal{J}(0,0 ; v(\cdot)) \geq \lambda \mathbb{E} \int_{0}^{T}|v(s)|^{2} d s=\lambda \mathbb{E} \int_{t}^{T}|u(s)|^{2} d s .
$$

Thus, $u(\cdot) \mapsto \mathcal{J}(t, 0 ; u(\cdot))$ is uniformly convex for any given $t \in[0, T)$. Let $\bar{u}(\cdot) \in \mathcal{U}[t, T]$ be an optimal control for $(t, \eta)$.

We can rewrite the cost functional as follows:

$$
\begin{aligned}
\mathcal{J}(t, \eta ; u(\cdot)) & \\
= & \left\langle G\left(\widehat{\Gamma}_{t} \eta+\widehat{\Xi}_{t} u\right), \widehat{\Gamma}_{t} \eta+\widehat{\Xi}_{t} u\right\rangle+\left\langle Q\left(\Gamma_{t} \eta+\Xi_{t} u\right), \Gamma_{t} \eta+\Xi_{t} u\right\rangle+\langle R u, u\rangle \\
= & \left\langle\left(\widehat{\Xi}_{t}^{*} G \widehat{\Xi}_{t}+\Xi_{t}^{*} Q \Xi_{t}+R\right) u, u\right\rangle+2\left\langle\left(\widehat{\Xi}_{t}^{*} G \widehat{\Gamma}_{t}+\Xi_{t}^{*} Q \Gamma_{t}\right) \eta, u\right\rangle \\
& +\left\langle\left(\widehat{\Gamma}_{t}^{*} G \widehat{\Gamma}_{t}+\Gamma_{t}^{*} Q \Gamma_{t}\right) \eta, \eta\right\rangle \\
= & \mathcal{J}(t, \eta ; 0)+\mathcal{J}(t, 0 ; u(\cdot))+2 \int_{t}^{T}\left\langle\left(\widehat{\Xi}_{t}^{*} G \widehat{\Gamma}_{t}+\Xi_{t}^{*} Q \Gamma_{t}\right) \eta, u\right\rangle d s \\
\geq & \lambda \mathbb{E} \int_{t}^{T}|\bar{u}(s)|^{2} d s+\mathcal{J}(t, \eta ; 0)-\frac{\lambda}{2} \mathbb{E} \int_{t}^{T}|\bar{u}(s)|^{2} d s-\frac{1}{2 \lambda} \mathbb{E} \int_{t}^{T}\left|\left(\widehat{\Xi}_{t}^{*} G \widehat{\Gamma}_{t}+\Xi_{t}^{*} Q \Gamma_{t}\right) \eta\right|^{2} d s \\
\geq & \frac{\lambda}{2} \mathbb{E} \int_{t}^{T}|\bar{u}(s)|^{2} d s+\mathcal{J}(t, \eta ; 0)-\frac{1}{2 \lambda} \mathbb{E} \int_{t}^{T}\left|\left(\widehat{\Xi}_{t}^{*} G \widehat{\Gamma}_{t}+\Xi_{t}^{*} Q \Gamma_{t}\right) \eta\right|^{2} d s .
\end{aligned}
$$

This implies that $u(\cdot) \mapsto \mathcal{J}(t, \eta ; u(\cdot))$ is coercivity. Clearly, $u(\cdot) \mapsto \mathcal{J}(t, \eta ; u(\cdot))$ continuous and convex. Consequently, it has a unique minimizer.

Moreover, (7.65) implies that

$$
V(t, \eta) \geq \mathcal{J}(t, \eta ; 0)-\frac{1}{2 \lambda} \mathbb{E} \int_{t}^{T}\left|\left(\widehat{\Xi}_{t}^{*} G \widehat{\Gamma}_{t}+\Xi_{t}^{*} Q \Gamma_{t}\right) \eta\right|^{2} d s .
$$

Since the functions on the right-hand side of (7.66) are quadratic in $x$ and continuous in $t$, we get (3.26). 


\subsection{Proof of Proposition 3.5}

Proof of Proposition 3.5: Let $\Theta(\cdot) \in L^{2}(0, T ; \mathcal{L}(U ; H))$ and $P(\cdot)$ be the solution to (3.17). For any $u(\cdot) \in \mathcal{U}[0, T]$, let $x_{0}(\cdot)$ be the solution to

$$
\left\{\begin{array}{l}
d x_{0}=\left[\left(A+A_{1}+B \Theta\right) x_{0}+B u\right] d s+\left[(C+D \Theta) x_{0}+D u\right] d W(s) \text { in }(0, T], \\
x_{0}(0)=0 .
\end{array}\right.
$$

It follows from (2.9) and Lemma 3.5 that

$$
\lambda \mathbb{E} \int_{0}^{T}\left|\Theta x_{0}+u\right|^{2} d s \leq \mathcal{J}\left(0,0 ; \Theta(\cdot) x_{0}(\cdot)+u(\cdot)\right)=\mathbb{E} \int_{0}^{T}\left[2\left\langle(L+K \Theta) x_{0}, u\right\rangle+\langle K u, u\rangle\right] d s .
$$

Consequently, for any $u(\cdot) \in \mathcal{U}[0, T]$, it holds that

$$
\mathbb{E} \int_{0}^{T}\left\{2\left\langle[L+(K-\lambda I) \Theta] x_{0}, u\right\rangle+\langle(K-\lambda I) u, u\rangle\right\} d s=\lambda \mathbb{E} \int_{0}^{T}\left|\Theta(s) x_{0}(s)\right|^{2} d s \geq 0
$$

Fix any $u_{0} \in U$, take $u(s)=u_{0} \chi_{[t, t+h]}(s)$, with $0 \leq t<t+h \leq T$. Then

$$
\left|x_{0}\right|_{C_{\mathbb{F}}\left([0, T] ; L^{2}(\Omega ; H)\right)} \leq \mathcal{C}|u|_{L_{\mathbb{F}}^{2}(0, T ; U)} \leq \mathcal{C} \sqrt{h}\left|u_{0}\right|_{U}
$$

Dividing both sides of (7.67) by $h$ and letting $h \rightarrow 0$, noting (7.68), we obtain

$$
\left\langle(K(s)-\lambda I) u_{0}, u_{0}\right\rangle \geq 0, \quad \text { a.e. } \quad s \in[0, T], \quad \forall u_{0} \in U .
$$

This gives the first inequality in (3.27). To prove the second one, for any $(t, \eta) \in[0, T) \times H$ and $u(\cdot) \in \mathcal{U}[t, T]$, let $x_{1}(\cdot)$ be the solution to

$$
\left\{\begin{array}{l}
d x_{1}=\left[\left(A+A_{1}+B \Theta\right) x_{1}+B u\right] d s+\left[(C+D \Theta) x_{1}+D u\right] d W(s) \quad \text { in }(t, T], \\
x_{1}(t)=\eta
\end{array}\right.
$$

It follows from Proposition 3.4 and Lemma 3.5 that

$$
\begin{aligned}
& \alpha|x|^{2} \leq V(t, \eta) \leq \mathcal{J}\left(t, \eta ; \Theta(\cdot) x_{1}(\cdot)+u(\cdot)\right) \\
& \left.=\langle P(t) \eta, \eta\rangle+\mathbb{E} \int_{t}^{T}\left[2\left\langle(L+K \Theta) x_{1}, u\right\rangle+K u, u\right\rangle\right] d s .
\end{aligned}
$$

In particular, by taking $u(\cdot)=0$ in the above, we obtain

$$
\langle P(t) \eta, \eta\rangle \geq \alpha|x|^{2}, \quad \forall(t, \eta) \in[0, T] \times H
$$

which gives the second inequality. 


\subsection{Proof of Lemmas 3.6 and 3.7}

Proof of Lemma 3.6. Similar to the proof of Proposition [3.1, one can show the existence of a unique solution $P \in C_{\mathcal{S}}([0, T] ; \mathcal{S}(H))$ to $(\underline{3.28})$. Let $\tilde{x}(\cdot)$ be the solution to the following SEE:

$$
\left\{\begin{array}{l}
d \tilde{x}=(A+\widetilde{A}) \tilde{x} d t+\widetilde{C} \tilde{x} d W(t) \quad \text { in }(t, T] \\
\tilde{x}(t)=\eta \in H
\end{array}\right.
$$

Clearly this equation admits a unique solution. By Itô's formula and Proposition 3.2.

$$
\langle\widetilde{P} \eta, \eta\rangle=\langle\widetilde{G} \tilde{x}(T), \tilde{x}(T)\rangle+\int_{t}^{T}\langle\widetilde{Q}(s) \tilde{x}(s), \tilde{x}(s)\rangle d s
$$

This, together with (3.29), implies that $P(\cdot) \in C\left([t, T] ; \overline{\mathbb{S}_{+}}(H)\right)$.

Proof of Lemma 3.7) Let $\Theta(\cdot) \in L^{2}(t, T ; \mathcal{L}(H ; U))$. Define a bounded linear operator $\mathfrak{L}: \mathcal{U}[t, T] \rightarrow \mathcal{U}[t, T]$ by

$$
\mathfrak{L} u=u-\Theta x .
$$

Then $\mathfrak{L}$ is bijective and its inverse $\mathfrak{L}^{-1}$ is given by

$$
\mathfrak{L}^{-1} u=u+\Theta \widetilde{x}
$$

where $\widetilde{x}(\cdot)$ is the solution to

$$
\left\{\begin{array}{l}
d \tilde{x}=\left[\left(A+A_{1}+B \Theta\right) \widetilde{x}+B u\right] d s+[(C+D \Theta) \widetilde{x}+D u] d W(s) \quad \text { in }(t, T] \\
\widetilde{x}(t)=0
\end{array}\right.
$$

By the bounded inverse theorem, $\mathfrak{L}^{-1}$ is bounded with norm $\left|\mathfrak{L}^{-1}\right|_{\mathcal{L}(\mathcal{U}[t, T])}>0$. Thus,

$$
\begin{aligned}
\mathbb{E} \int_{t}^{T}|u(s)|_{U}^{2} d s & =\mathbb{E} \int_{t}^{T}\left|\left(\mathfrak{L}^{-1} \mathfrak{L} u\right)(s)\right|_{U}^{2} d s \leq\left|\mathfrak{L}^{-1}\right| \mathbb{E} \int_{t}^{T}|(\mathfrak{L} u)(s)|_{U}^{2} d s \\
& =\left|\mathfrak{L}^{-1}\right|_{\mathcal{L}(\mathcal{U}[t, T])} \mathbb{E} \int_{t}^{T}|u(s)-\Theta(s) x(s)|_{U}^{2} d s, \quad \forall u(\cdot) \in \mathcal{U}[t, T]
\end{aligned}
$$

which implies (3.30) with $c_{0}=\left|\mathfrak{L}^{-1}\right|_{\mathcal{L}(\mathcal{U}[t, T])}^{-1}$.

\section{References}

[1] N. U. Ahmed, Stochastic control on Hilbert space for linear evolution equations with random operator-valued coefficients. SIAM J. Control Optim. 19 (1981), 401-430.

[2] B. D. O. Anderson and J. B. Moore, Optimal Control: Linear Quadratic Methods. Prentice Hall, Englewood Cliffs, NJ, 1989.

[3] R. Bellman, I. Glicksberg and O. Gross, Some Aspects of the mathematical Theory of Control Processes. RAND Corporation, Santa Monica, CA, 1958. 
[4] A. Bensoussan, G. Da Prato, M. C. Delfour and S. K. Mitter, Representation and control of infinite-dimensional systems. Vol. II. Systems \& Control: Foundations \& Applications. Birkhäuser Boston, Inc., Boston, MA, 1993.

[5] F. J. Beutler, The operator theory of the pseudo-inverse. II. Unbounded operators with arbitrary range. J. Math. Anal. Appl. 10(1965), 471-493.

[6] J.-M. Bismut, Contrôle des systèmes linéaires quadratiques: applications de l'intégrale stochastique. pp. 180-264, Lecture Notes in Math., 649, Springer, Berlin, 1978.

[7] R. A. Carmona and B. Rozovskii, eds. Stochastic Partial Differential Equations: Six Perspectives. Math. Surveys Monogr. 64, AMS, Providence, RI, 1999.

[8] S. Chen, X. Li and X. Zhou, Stochastic linear quadratic regulators with indefinite control weight costs. SIAM J. Control Optim. 36 (1998), 1685-1702.

[9] S. Chen and X. Y. Zhou, Stochastic linear quadratic regulators with indefinite control weight costs. II, SIAM J. Control Optim. 39 (2000), 1065-1081.

[10] R. F. Curtain and A. Ichikawa, The separation principle for stochastic evolution equations. SIAM J. Control Optim. 15 (1977), 367-383.

[11] R. F. Curtain, Estimation and stochastic control for linear infinite-dimensional systems. Probabilistic analysis and related topics, Vol. 1, pp. 45-86. Academic Press, New York, 1978.

[12] G. Da Prato and J. Zabczyk, Stochastic Equations in Infinite Dimensions. Cambridge University Press, Cambridge, 1992.

[13] G. Guatteri and G. Tessitore, On the backward stochastic Riccati equation in infinite dimensions. SIAM J. Control Optim. 44 (2005), 159-194.

[14] G. Guatteri and G. Tessitore, Well posedness of operator valued backward stochastic Riccati equations in infinite dimensional spaces. SIAM J. Control Optim. 52 (2014), 3776-3806.

[15] M. Kohlmann and S. Tang, Multidimensional backward stochastic Riccati equations and applications. SIAM J. Control Optim. 41 (2003), 1696-1721.

[16] C. Hafizoglu, I. Lasiecka, T. Levajković, H. Mena and A. Tuffaha, The stochastic linear quadratic control problem with singular estimates. SIAM J. Control Optim. 55 (2017), 595-626.

[17] Y. Hu and X. Y. Zhou, Indefinite stochastic Riccati equations, SIAM J. Control Optim. 42 (2003), 123-137.

[18] V. N. Kolokoltsov, Semiclassical analysis for diffusions and stochastic processes. Lecture Notes in Mathematics, 1724, Springer-Verlag, Berlin, 2000. 
[19] P. Kotelenez, Stochastic ordinary and stochastic partial differential equations. Transition from microscopic to macroscopic equations. Stochastic Modelling and Applied Probability, 58. Springer, New York, 2008.

[20] I. Lasiecka and R. Triggiani, Differential and algebraic Riccati equations with applications to boundary/point controlproblems: continuous theory and approximation theory. Lecture Notes in Control \& Inform. Sci., Vo1.164, Springer-Verlag, Berlin, 1991.

[21] X. Li, X. Zhou and A. E. B. Lim, Dynamic mean-variance portfolio selection with noshorting constraints. SIAM J. Control Optim. 40 (2002), 1540-1555.

[22] Q. Lü, T. Wang and X. Zhang, Characterization of Optimal Feedback for Stochastic Linear Quadratic Control Problems. Probab. Uncertain. Quant. Risk 2 (2017), Paper No. 11, 20 pp.

[23] Q. Lü and X. Zhang, General Pontryagin-Type Stochastic Maximum Principle and Backward Stochastic Evolution Equations in Infinite Dimensions. Springer Briefs in Mathematics. Springer, Cham, 2014.

[24] Q. Lü and X. Zhang, Transposition method for backward stochastic evolution equations revisited, and its application. Math. Control Relat. Fields. 5 (2015), 529-555.

[25] N. I. Mahmudov and M. A. McKibben, On backward stochastic evolution equations in Hilbert spaces and optimal control. Nonlinear Anal. 67 (2007), 1260-1274.

[26] Z. Qian and X. Y. Zhou, Existence of solutions to a class of indefinite stochastic Riccati equations, SIAM J. Control Optim. 51 (2013), 221-229.

[27] M. Rami, X. Chen, J. B. Moore and X. Zhou, Solvability and asymptotic behavior of generalized Riccati equations arising in indefinite stochastic $L Q$ controls. IEEE Trans. Automat. Control 46 (2001), 428-440.

[28] J. Sun, X. Li and J. Yong, Open-loop and closed-loop solvabilities for stochastic linear quadratic optimal control problems. SIAM J. Control Optim. 54 (2016), 2274-2308.

[29] J. Sun and J. Yong, Linear Quadratic Stochastic Differential Games: Open-Loop and Closed-Loop Saddle Points, SIAM J. Control Optim. 52 (2014), 4082-4121.

[30] J. Sun and J. Yong, Stochastic linear quadratic optimal control problems in infinite horizon. Appl. Math. Optim. 78 (2018), 145-183.

[31] S. Tang, General linear quadratic optimal stochastic control problems with random coefficients: linear stochastic Hamilton systems and backward stochastic Riccati equations. SIAM J. Control Optim. 42 (2003), 53-75.

[32] G. Tessitore, Some remarks on the Riccati equation arising in an optimal control problem with state- and control-dependent noise. SIAM J. Control Optim. 30 (1992), 717-744.

[33] G. Tessitore, Linear quadratic optimal control for a stochastic system with control on the boundary and hyperbolic dynamics. J. Math. Systems Estim. Control 2 (1992), 453-482. 
[34] J. M. A. M. van Neerven, M. C. Veraar and L. Weis, Stochastic integration in UMD Banach spaces. Ann. Probab. 35 (2007), 1438-1478.

[35] J. M. A. M. van Neerven, $\gamma$-radonifying operators - a survey. The AMSI-ANU Workshop on Spectral Theory and Harmonic Analysis, 1-61. Proc. Centre Math. Appl. Austral. Nat. Univ., 44, Austral. Nat. Univ., Canberra, 2010.

[36] W. M. Wonham, On a matrix Riccati equation of stochastic control, SIAM J. Control Optim. 6 (1968), 312-326.

[37] J. Yong and X. Y. Zhou, Stochastic Controls: Hamiltonian Systems and HJB Equations, Springer-Verlag, New York, 1999.

[38] Z. Yu, An optimal feedback control-strategy pair for zero-sum linear-quadratic stochastic differential game: the Riccati equation approach. SIAM J. Control Optim. 53 (2015), 2141-2167. 